\title{
Epidemiology of physical inactivity, sedentary behaviors, and unhealthy eating habits among Brazilian adolescents: a systematic review
}

\author{
Epidemiologia da inatividade física, comportamentos sedentários \\ e hábitos alimentares não-saudáveis em adolescentes brasileiros: \\ uma revisão sistemática
}

Valter Cordeiro Barbosa Filho ${ }^{1}$

Wagner de Campos ${ }^{1}$

Adair da Silva Lopes ${ }^{2}$

${ }^{1}$ Departamento de Educação Física, Universidade Federal do Paraná. R. Coração de Maria 92/Br 116/Km 95, Jardim Botânico. 80.215-370 Curitiba PR Brasil.valtercbf@gmail.com ${ }^{2}$ Centro de Desportos,

Departamento de Educação Física, Universidade Federal de Santa Catarina.
Abstract This systematic review analyzed the prevalence of physical inactivity, sedentary behaviors and unhealthy eating habits among Brazilian adolescents. Searches were conducted in five databases (Lilacs, SciELO, Medline, Web of Science, and Google Scholar) and in the references cited in the articles retrieved. The literature search yielded 5,872 potentially relevant titles and a total of 69 studies met all the inclusion criteria. The risk behavior most often evaluated was physical inactivity (48/69; 69.6\%), and its prevalence rate ranged from $2.3 \%$ to $93.5 \%$. Twenty-eight studies estimated the prevalence of physical inactivity at over $50 \%$. Most studies observed the prevalence of greater physical inactivity among girls. The prevalence of sedentary behaviors (lengthy screen time or TV use) was also frequently over 50\%. Several variables were used to identify unhealthy eating habits, and some criteria/studies have indicated unhealthy eating habit estimates at close to $100 \%$ among adolescents. In conclusion, the estimates of these risk behaviors among Brazilians adolescents were very close to or even greater than those found in developed countries in several studies analyzed in this review.

Key words Risk factors, Sedentary lifestyle, Eating behavior, Adolescent, Review
Resumo Esta revisão sistemática analisou a prevalência de inatividade física, comportamentos sedentários e hábitos alimentares não-saudáveis em adolescentes brasileiros. Buscas foram realizadas em cinco bases de dados (Lilacs, SciELO, Medline, Web of Science e o Google Scholar) e nas referências dos artigos recuperados. A pesquisa bibliográfica rendeu 5.872 títulos potencialmente relevantes; 69 estudos preencheram todos os critérios de inclusão. O comportamento de risco mais frequentemente avaliado foi a inatividade física (48/69; 69,6\%), e sua taxa de prevalência variou de 2,3\% a 93,5\%. Vinte e oito estudos estimaram taxas de inatividade física acima de 50\%. A maioria dos estudos indicou taxas de inatividade física superiores entre meninas. As prevalências de comportamentos sedentários (elevado tempo de tela ou usando TV) também esteviveram, frequentemente, acima de 50\%. Diversas variáveis foram utilizadas para a definição de hábitos alimentares não saudáveis, e alguns critérios têm indicado estimativas próximas a 100\%. Em conclusão, diversos estudos analisados nesta revisão apontaram estimativas de comportamentos de risco em adolescentes brasileiros muito próximas, ou até superiores, às obtidas em países desenvolvidos.

Palavras-chave Fatores de risco, Estilo de vida sedentário, Comportamento alimentar, Jovem, Revisão 


\section{Introduction}

Chronic non-communicable diseases (NCDs) are the leading causes of death globally $(60 \%$ of all deaths), killing more people each year than all other causes combined ${ }^{1}$. Alarming estimates suggest that NCD deaths will increase $15 \%$ globally between 2010 and $2020^{2}$. Among the NCD-related risk factors, the World Health Organization $(\mathrm{WHO})^{1-3}$ highlights two behaviors that are pervasive aspects of economic transition, rapid urbanization and a 21st-century lifestyle: physical inactivity and unhealthy food habits.

Physical inactivity has been identified as the fourth leading risk factor for global mortality ( $6 \%$ of deaths globally). Physical inactivity overcoming even overweight and obesity $(5 \%)$ as global risk factor for mortality ${ }^{4}$. The available data also indicate that unhealthy food habits are related strongly to the emergence of NCDs and NCD-related mortality. Approximately 1.7 million $(2.8 \%)$ deaths worldwide are attributable to low fruit and vegetable consumption ${ }^{5}$. Likewise, low consumption of fruit and vegetables has been directly related to the risk for cardiovascular diseases, stomach cancer and colorectal cancer ${ }^{5}$. Thus, unhealthy food habits and physical inactivity contribute substantially to the global burden of disease, death, and disability,

Morbidity and mortality studies have also focused on another NCD behavioral risk factor: sedentary behavior (i.e., too much sitting, as distinct from too little exercise) $)^{7}$. Recent studies had shown a positive and independent relationship between sedentary behavior and mortality among adults $^{7-9}$. There is also evidence to suggest that sedentary behavior has a positive relationship with adverse health outcomes in adults (e.g., cardiovascular diseases and diabetes $)^{10}$, including whether the sedentary behavior was adopted during childhood ${ }^{11}$. Thus, sedentary behavior is an important area of study and is in need of further development.

Although physical inactivity, unhealthy food habits, and sedentary behaviors are not the major behavioral risk factors for morbidity and mortality during adolescence ${ }^{5,12}$, these behaviors are frequently interrelated. Additionally, they are often established during childhood and adolescence and track into adult life ${ }^{12}$, having a considerable effect on health at later ages. ${ }^{5}$ Therefore, the $\mathrm{WHO}^{1}$ has considered monitoring these behaviors during adolescence a priority, and studies have been conducted in adolescents from several countries ${ }^{12-14}$.
As a consequence, many local and regional epidemiological studies have been conducted in Brazil concerning physical inactivity, unhealthy food habits, and/or sedentary behaviors among adolescents. To contribute to the development of public policies for health promotion in Brazil, a recent survey involving adolescents from all 26 Brazilian state capitals and the Federal District ${ }^{15}$ was conducted with the primary purpose of monitoring risk behaviors among Brazilian adolescents.

The main objective of this systematic review was to analyze the prevalence of physical inactivity, sedentary behaviors, and unhealthy food habits in studies including Brazilian adolescents (1019 years old). This review contributes to the understanding of these behavioral risk factors in Brazilian adolescents who are and who are not included in national studies (i.e., teenagers from small towns) and identifies the primary behavioral risk factors in Brazilian youth. As the patterns of these behavioral risk factors may differ according to gender ${ }^{6}$, this review had a secondary objective of analyzing differences in these behavioral risk factors between genders.

\section{Methods}

The literature search was performed in June 2011 using the following electronic databases: LILACS, SciELO, PubMed, Web of Science, and the Google Scholar portal. The year and language of publication of the manuscripts were not limited.

Search strategies were built around three groups of keywords: risk behaviors, sample type and nationality. The keywords of the risk behaviors were stratified into four subgroups, featuring the reviewed behavioral outcomes: (i) general terms of risk factors (adolescent behavio* OR adolescent health OR cardiovascular health OR cardiovascular diseas ${ }^{*}$ OR health behavio* OR lifestyle OR risky behavio* OR risk factor ${ }^{\star}$ ); (ii) physical inactivity (exercise OR motor activity OR physical activity OR physical exercise OR physical fitness OR physical inactivity OR inactivity); (iii) sedentary behaviors (computer use OR screen viewing OR sedentary behavio* OR sedentary lifestyle OR television OR sitting time); and (iv) unhealthy food habits (diet OR eating habit OR feeding behavio* OR food consumption OR food intake OR health food OR nutrition OR unhealthy food). Each subgroup was used in combination with keywords for sample type (youth OR teen ${ }^{\star}$ OR adolescent ${ }^{\star}$ OR ado- 
lescence OR child ${ }^{\star}$ ) and nationality (Brazil ${ }^{\star}$ OR Brazilian) to locate potentially relevant studies. The Boolean operator "AND" was used for combinations between the groups. The article search was conducted with keywords in English and Portuguese.

The first author of this study (VCBF) selected articles using a systematic method. He first read the article titles. Then, he read abstracts and used the inclusion criteria to analyze the articles. After examining the abstracts, all of the full-text the articles were obtained and included if they met the inclusion criteria. The references for all selected papers were examined to identify other publications that should be reviewed.

The following inclusion criteria were adopted in this review: (i) articles published in peer-reviewed journals that were original research; (ii) samples included Brazilian adolescents aged 1019 years (or a mean age within these ranges) or a sample comprising other age years, because the data had been presented separately; (iii) observational studies showing the prevalence of at least one of the behavioral risk factors included in this review, regardless of whether the study dealt with this behavior as an exposure or a response variable; (iv) studies using questionnaires or structured interviews as methods for behavior assessment; and (v) to be a school- or populationbased survey with information about the methodological procedures of representation of the target population (i.e., random sampling).

Theses, dissertations and monographs were not included because it was impractical to systematically search them. In cases of duplicates (two or more studies with the same sample), the most recent or the most complete publication was used, and the other publications were excluded.

The following data were extracted: local of the study, sample type, sample size, percentage of girls, age years of adolescents, type and administration mode of instrument, recall time, and definition of the behavioral risk factors. For an additional description of studies, the Human Development Index (HDI; base year 2000) of the local survey was considered and presented in three categories: low (HDI $<0.600$ ), middle (HDI between 0.600 and 0.799$)$ and high (HDI $\geq 0.800)$.

Studies including the physical inactivity prevalence were organized into three groups. The first group included studies considering the cutoff point adopted in the Global School-based Student Health Survey (GSHS; less than 300 minutes/week of physical activity or moderate-tovigorous physical activity specifically $)^{13,14}$, which was an international survey that identified the physical inactivity prevalence among adolescents from several countries. The second group of studies used other physical inactivity definitions adopted in the literature, while the third group consisted of studies that used the low frequency of participation in physical activities and sports as an indicator of physical inactivity.

The prevalence of sedentary behaviors among Brazilian adolescents has focused on two important outcomes. The first is related to high screen time use (e.g., playing video games or using a computer) during the day. The second study group focused exclusively on high TV time use.

The studies evaluating the prevalence of unhealthy food habits treated food consumption in two ways: the low consumption of healthy foods (e.g., fruits and vegetables) or the high consumption of unhealthy foods (e.g., sweets or soft drinks). Data were also included identifying the prevalence of adolescents who did not meet the WHO recommendations for healthy diet or who had a high fat-rich foods intake (e.g., processed meats and fried chicken), or a low fiber-rich foods intake (e.g., fruits, beans, and rice).

The outcome prevalence and its respective $95 \%$ confidence interval ( $95 \% \mathrm{CI}$ ) were presented with the total sample and by gender. The $95 \%$ CI was directly from articles ${ }^{15-27}$, whenever possible, or calculated using the statistics program Stata 10.0 (Stata Corp., College Station, United States) using the 'cii' command (95\% CI exact for binomial distribution). The included articles were organized in tables according to the criteria used to identify the behavioral risk factor and in alphabetical order considering the location of study.

\section{Results}

\section{Literature search}

The literature search yielded 5,872 potentially relevant articles. After reading all titles, 821 were selected based on the inclusion criteria of this review. One hundred and thirty-nine studies were selected for a full-text reading. Of these studies, seventy-one (51.1\%) were excluded for the following reasons: (i) 27 studies used the convenience sample selection method; (ii) 23 studies did not present the variables of interest of this review; (iii) 15 studies presented replicated data; and (iv) 6 studies not included adolescents. Thus, 68 studies met all inclusion criteria and were presented in this review. Additionally, one study ${ }^{28}$ 
was identified by searching the article reference lists and included. Thus, a total of 69 studies were included in this review.

\section{Study characteristics}

Table 1 shows the main characteristics of the 69 studies included in this review. Most studies

Table 1. Characteristics of studies included in this review $(n=69)$.

\begin{tabular}{|c|c|c|}
\hline Characteristics & Reference & n [\% of 69 studies] \\
\hline \multicolumn{3}{|l|}{ Geographical region $^{*}$} \\
\hline Northeastern & {$[16,30,31,32,33,34,35,36,37,38,39,40,41]$} & $13[18.8]$ \\
\hline Central-Western & {$[42,43]$} & $2[2.9]$ \\
\hline Southeastern & {$[24,25,29,44,45,46,47,48,49,50,51,52,53,54,55,56,57,58]$} & $18[26.1]$ \\
\hline Southern & $\begin{array}{l}{[17,18,19,20,21,22,23,59,60,61,62,63,64,65,66,67,68,69,70,71,72,} \\
73,74,75,76,77,78,79,80,81,82,83]\end{array}$ & $32[46.4]$ \\
\hline Two or more regions & {$[15,26,27,28]$} & $4[5.8]$ \\
\hline \multicolumn{3}{|l|}{$\mathrm{HDI}^{* *}$} \\
\hline$<0.600$ & {$[34]$} & $1[1.4]$ \\
\hline $0.600-0.799$ & {$\left[16,28,27^{* * *}, 29,30,32,33,35,36,38,39,41,44,60,61\right]$} & $15[21.7]$ \\
\hline$\geq 0.800$ & $\begin{array}{l}{\left[15,17,18,19,20,21,22,23,24,25,26,27^{* * *}, 31,37,40,42,43,45,46,47\right.} \\
48,49,50,51,52,53,54,55,56,57,58,59,62,63,64,65,66,67,68,69 \\
70,71,72,73,74,75,76,77,78,79,80,81,82,83]\end{array}$ & $54[78.3]$ \\
\hline \multicolumn{3}{|c|}{ 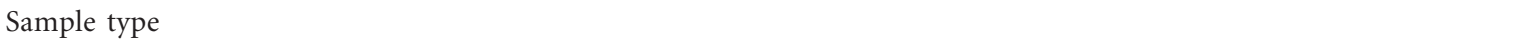 } \\
\hline $\begin{array}{l}\text { Population-based } \\
\text { School-based }\end{array}$ & {$[18,22,27,28,31,47,49,55,57,59,63,64,67,68,69,72,73,75,82]$} & $18[26.1]$ \\
\hline Public and private schools & $\begin{array}{l}{[15,20,21,26,30,32,36,38,39,43,44,46,48,50,58,60,62,65,66,70,71,} \\
74,77,81]\end{array}$ & $24[34.8]$ \\
\hline Public schools only & $\begin{array}{l}{[16,17,19,23,24,25,27,29,34,35,37,40,41,42,45,51,52,53,54,56,61,} \\
76,78,79,80,83]\end{array}$ & $26[37.7]$ \\
\hline Private schools only & {$[33]$} & $1[1.4]$ \\
\hline \multicolumn{3}{|l|}{ Sample size } \\
\hline$<300$ & {$[29,34]$} & $2[2.9]$ \\
\hline $300-499$ & {$[31,35,45,50,53,54,56,61]$} & $8[11.6]$ \\
\hline $500-999$ & {$[36,38,40,44,47,51,55,57,59,60,62,64,69,70,71,72,73,76,78,82]$} & $20[29.0]$ \\
\hline $1000-2,000$ & {$[17,19,20,21,24,28,30,37,41,43,46,48,49,52,65,66,67,68,74]$} & $19[27.5]$ \\
\hline$\geq 2,000$ & {$[15,16,18,22,23,25,26,27,32,33,39,42,58,63,75,77,79,80,81,83]$} & $20[29.0]$ \\
\hline \multicolumn{3}{|c|}{ Range of age included } \\
\hline Adolescents only (10-19 years) & $\begin{array}{l}{[17,18,19,20,22,23,25,27,29,31,32,33,35,39,40,43,45,46,50,51,53,} \\
54,55,56,58,59,61,62,63,64,65,66,67,68,69,70,72,73,74,75,76, \\
77,78,79,80,81,82]\end{array}$ & $47[68.2]$ \\
\hline Also children $(<10$ years $)$ & {$[21,30,36,37,38,44,48,52,71]$} & $9[13.0]$ \\
\hline Also adults (>19 years) & {$[15,16,24,26,27,28,34,41,42,47,49,57,60]$} & $13[18.8]$ \\
\hline \multicolumn{3}{|l|}{ Mode of administration } \\
\hline Self & $\begin{array}{l}{[15,16,17,19,20,24,23,25,26,27,32,33,34,35,36,37,38,39,40,41,42,} \\
43,45,47,48,50,51,52,58,60,61,65,66,68,71,74,76,77,78,79, \\
80,81,83]\end{array}$ & $43[62.3]$ \\
\hline Interviewer & $\begin{array}{l}{[18,21,22,28,29,30,31,44,46,49,53,54,55,56,57,59,62,63,64,67,69} \\
70,72,73,75,82]\end{array}$ & $26[37.7]$ \\
\hline \multicolumn{3}{|l|}{ Years of survey } \\
\hline 1995-1999 & {$[28,42,45,47,58,67,81]$} & $7[10.1]$ \\
\hline $2000-2004$ & $\begin{array}{l}{[22,23,24,30,31,33,35,49,51,53,54,55,56,57,63,66,69,70,72,73,75} \\
77,79,80,82,83]\end{array}$ & $26[37.7]$ \\
\hline $2005-2006$ & {$[16,17,19,20,25,27,29,32,36,38,41,44,52,60,61,64,68,78]$} & $18[26.1]$ \\
\hline 2007-2009 & {$[15,18,21,26,34,37,39,40,43,46,59,62,65]$} & $13[18.8]$ \\
\hline Not mentioned & {$[48,50,71,74,76]$} & $5[7.3]$ \\
\hline
\end{tabular}

"No study included in this review was conducted in the Northern Brazil. ** Human Development Index (HDI) was based on data from United Nations Development Programme for different cities and regions of Brazil, reference year 2000. ${ }^{* * *}$ Nahas et al. ${ }^{27}$ included adolescents from Recife $(\mathrm{HDI}=0.797)$ and Florianópolis $(\mathrm{HDI}=0.875)$, and thus was cited twice. 
were conducted in Southern Brazil (46.4\%), and no study included in this review was performed with adolescents from Northern Brazil. Most studies were conducted in sites with a high HDI (78.3\%). Only one study (1.4\%) was performed in a city with a low HDI. Most studies (73.9\%) were school-based surveys, and a predominance of studies included only adolescents from public schools (37.7\%). The sample size of included studies varied from $105^{29}$ to $60,973^{15,26}$ individuals. Several studies had sample sizes of 500-999 or more than 2,000 individuals (20 studies in each category). Finally, several studies included only adolescents in the sample $(68.2 \%)$, used self-reports to fill out of the questionnaires (62.3\%), and were conducted between 2005 and 2009 $(42.0 \%)$.

\section{Prevalence of physical inactivity}

Table 2 shows some characteristics of the studies evaluating the physical inactivity prevalence. A total of 48 studies included this outcome, and the physical inactivity prevalence ranged from $2.3 \%$ (95\% CI: $1.3-3.8)^{60}$ to $93.5 \%$ (95\% CI: 92.0-94.8) ${ }^{30}$. Only four studies $(8.3 \%)^{26,46,51,60}$ showed a physical inactivity prevalence of less than $20 \%$, while 28 studies $(58.3 \%)^{15,17-21,24,25,28,30-35,44-47,61-69}$ estimated a physical inactivity prevalence above $50 \%$.

Nineteen studies used the GSHS physical inactivity definition, and the lowest physical inactivity prevalence was found in Piedade, SP (18.1\%; 95\% CI: 11.3-26.8 $)^{29}$, and the highest was found in Curitiba, PR (85.5\%; 95\% CI: 83.6-87.2) ${ }^{17}$. Of the 19 studies, thirteen $(68.4 \%)^{15,17-20,24,25,31,44,61-64}$

Table 2. Description of the prevalence of physical inactivity (\%) among Brazilian adolescents and its respective $95 \%$ confidence interval (95\% CI, entire sample and by gender) of each study included in this review according to local and year of survey, sample, age, instrument, and definition of physical inactivity.

\begin{tabular}{|c|c|c|c|c|c|c|c|}
\hline \multirow[b]{2}{*}{ Local (survey year) } & \multirow{2}{*}{$\begin{array}{c}\text { Sample } \\
(\% \text { of girls })\end{array}$} & \multirow{2}{*}{$\begin{array}{c}\text { Age } \\
\text { (years) }\end{array}$} & \multirow[b]{2}{*}{ Instrument; recall time } & \multirow[b]{2}{*}{ Definition } & \multicolumn{3}{|c|}{ Prevalence \% (95\% CI) } \\
\hline & & & & & All & Boys & Girls \\
\hline \multicolumn{8}{|l|}{$\begin{array}{l}\text { Main physical } \\
\text { inactivity definitions }\end{array}$} \\
\hline $\begin{array}{l}\text { All state capital } \\
\text { cities }(2009)^{15}\end{array}$ & $\begin{array}{c}60,973 \\
(53.3)\end{array}$ & grade 8 & Q-GSHS; PR-W & $\begin{array}{c}<300 \mathrm{~min} . / \text { week } \\
\text { of MVPA }\end{array}$ & $\begin{array}{c}56.9 \\
(56.2-57.6)\end{array}$ & $\begin{array}{c}43.8 \\
(42.8-44.8)\end{array}$ & $\begin{array}{c}68.7 \\
(67.8-69.6)^{*}\end{array}$ \\
\hline $\begin{array}{l}\text { Curitiba, PR } \\
(2006)^{17}\end{array}$ & $\begin{array}{l}1,518 \\
(59.2)\end{array}$ & $14-18$ & Q-GSHS; HA-W & $\begin{array}{c}<300 \mathrm{~min} . / \text { week } \\
\text { of MVPA }\end{array}$ & $\begin{array}{c}85.5 \\
(83.6-87.2)\end{array}$ & $\begin{array}{c}77.7 \\
(74.2-80.9)\end{array}$ & $\begin{array}{c}90.9 \\
(88.8-92.7)^{*}\end{array}$ \\
\hline $\begin{array}{l}\text { Foz do Iguaçu, PR } \\
(2005)^{61}\end{array}$ & $\begin{array}{c}453 \\
(59.2)\end{array}$ & $15-18$ & Q-GSHS; PR-W & $\begin{array}{c}<300 \mathrm{~min} . / \text { week } \\
\text { of MVPA }\end{array}$ & $\begin{array}{c}77.0 \\
(72.9-80.8)\end{array}$ & $\begin{array}{c}77.8 \\
(71.1-83.6)\end{array}$ & $\begin{array}{c}76.5 \\
(70.1-81.4)\end{array}$ \\
\hline $\begin{array}{l}\text { João Pessoa, PB } \\
(2009)^{39}\end{array}$ & $\begin{array}{r}2,874 \\
(57.8)\end{array}$ & $14-19$ & $\begin{array}{c}\text { Own questionnaire; } \\
\text { PR-W }\end{array}$ & $\begin{array}{c}<300 \mathrm{~min} . / \text { week } \\
\text { of MVPA }\end{array}$ & $\begin{array}{c}49.8 \\
(46.9-52.7)\end{array}$ & $\begin{array}{c}33.7 \\
(31.0-36.4)\end{array}$ & $\begin{array}{c}61.5 \\
(59.1-63.9)^{*}\end{array}$ \\
\hline $\begin{array}{l}\text { Londrina, PR } \\
(2005)^{78}\end{array}$ & $\begin{array}{c}664 \\
(61.6)\end{array}$ & $15-18$ & IPAQ; PR-W & $\begin{array}{c}<300 \mathrm{~min} . / \text { week } \\
\text { of PA }\end{array}$ & $\begin{array}{c}39.2 \\
(35.4-43.1)\end{array}$ & $\begin{array}{c}33.3 \\
(27.4-39.4)\end{array}$ & $\begin{array}{c}42.8 \\
(37.9-47.8)\end{array}$ \\
\hline $\begin{array}{l}\text { Maringá, PR } \\
(2007)^{62}\end{array}$ & $\begin{array}{c}991 \\
(54.5)\end{array}$ & $14-18$ & IPAQ; PR-W & $\begin{array}{c}<300 \mathrm{~min} . / \text { week } \\
\text { of MVPA }\end{array}$ & $\begin{array}{c}56.9 \\
(53.8-60.0)\end{array}$ & $\begin{array}{c}55.7 \\
(50.9-60.3)\end{array}$ & $\begin{array}{c}57.9 \\
(53.7-62.2)^{*}\end{array}$ \\
\hline $\begin{array}{l}\text { Ouro Preto, MG } \\
(2006)^{44}\end{array}$ & $\begin{array}{c}780 \\
(52.6)\end{array}$ & $6-14$ & Own questionnaire; n.a. & $\begin{array}{c}<300 \mathrm{~min} . / \text { week } \\
\text { of PA }\end{array}$ & $\begin{array}{c}79.3 \\
(76.1-82.3)\end{array}$ & n.a. & n.a. \\
\hline $\begin{array}{l}\text { Pelotas, RS (2004- } \\
2005)^{63}\end{array}$ & $\begin{array}{l}4,452 \\
(49.2)\end{array}$ & $10-12$ & $\begin{array}{c}\text { Own questionnaire; } \\
\text { PR-W }\end{array}$ & $\begin{array}{c}<300 \mathrm{~min} . / \text { week } \\
\text { of PA }\end{array}$ & $\begin{array}{c}58.2 \\
(56.7-59.7)\end{array}$ & $\begin{array}{c}49.0 \\
(46.8-51.1)\end{array}$ & $\begin{array}{c}67.0 \\
(65.1-69.0)^{*}\end{array}$ \\
\hline Pelotas, RS $(2005)^{64}$ & $\begin{array}{c}857 \\
(52.0)\end{array}$ & $10-19$ & $\begin{array}{c}\text { Own questionnaire; } \\
\text { PR-W }\end{array}$ & $\begin{array}{c}<300 \mathrm{~min} . / \text { week } \\
\text { of MVPA }\end{array}$ & $\begin{array}{c}69.8 \\
(66.7-72.9)\end{array}$ & $\begin{array}{c}56.5 \\
(51.6-61.3)\end{array}$ & $\begin{array}{c}82.1 \\
(78.5-85.6)^{*}\end{array}$ \\
\hline Pelotas, RS $(2008)^{18}$ & $\begin{array}{l}4,325 \\
(51.0)\end{array}$ & $14-15$ & $\begin{array}{c}\text { Own questionnaire; } \\
\text { PR-W }\end{array}$ & $\begin{array}{c}<300 \mathrm{~min} . / \text { week } \\
\text { of PA }\end{array}$ & $\begin{array}{c}51.8 \\
(50.3-52.3)\end{array}$ & $\begin{array}{c}37.4 \\
(35.4-39.5)\end{array}$ & $\begin{array}{c}65.5 \\
(63.5-67.5)^{*}\end{array}$ \\
\hline $\begin{array}{l}\text { Pernambuco state } \\
(2006)^{19}\end{array}$ & $\begin{array}{l}4,210 \\
(59.8)\end{array}$ & $14-20$ & Q-GSHS; PR-W & $\begin{array}{c}<300 \mathrm{~min} . / \text { week } \\
\text { of MVPA }\end{array}$ & $\begin{array}{c}65.1 \\
(63.7-66.6)\end{array}$ & $\begin{array}{c}57.6 \\
(55.2-60.0)\end{array}$ & $\begin{array}{c}70.2 \\
(68.3-71.9)^{*}\end{array}$ \\
\hline $\begin{array}{l}\text { Piedade, SP } \\
(2005)^{29}\end{array}$ & $\begin{array}{c}105 \\
(63.0)\end{array}$ & $10-14$ & $\begin{array}{l}\text { Questionnaire proposed } \\
\text { by Florindo et al. } \\
\text { (2006); PR-Y }\end{array}$ & $\begin{array}{c}<300 \mathrm{~min} . / \text { week } \\
\text { of PA }\end{array}$ & $\begin{array}{c}18.1 \\
(11.3-26.8)\end{array}$ & n.a. & n.a. \\
\hline $\begin{array}{l}\text { Piracicaba, SP } \\
(2004)^{56}\end{array}$ & $\begin{array}{c}390 \\
(53.4)\end{array}$ & $10-17$ & $\begin{array}{c}\text { Questionnaire proposed } \\
\text { by Florindo et al. } \\
\text { (2006); PR-Y }\end{array}$ & $\begin{array}{c}<300 \mathrm{~min} . / \text { week } \\
\text { of PA }\end{array}$ & $\begin{array}{c}54.9 \\
(49.3-60.3)\end{array}$ & $\begin{array}{c}42.0 \\
(34.0-50.3)\end{array}$ & $\begin{array}{c}65.7 \\
(58.3-72.7)^{*}\end{array}$ \\
\hline
\end{tabular}




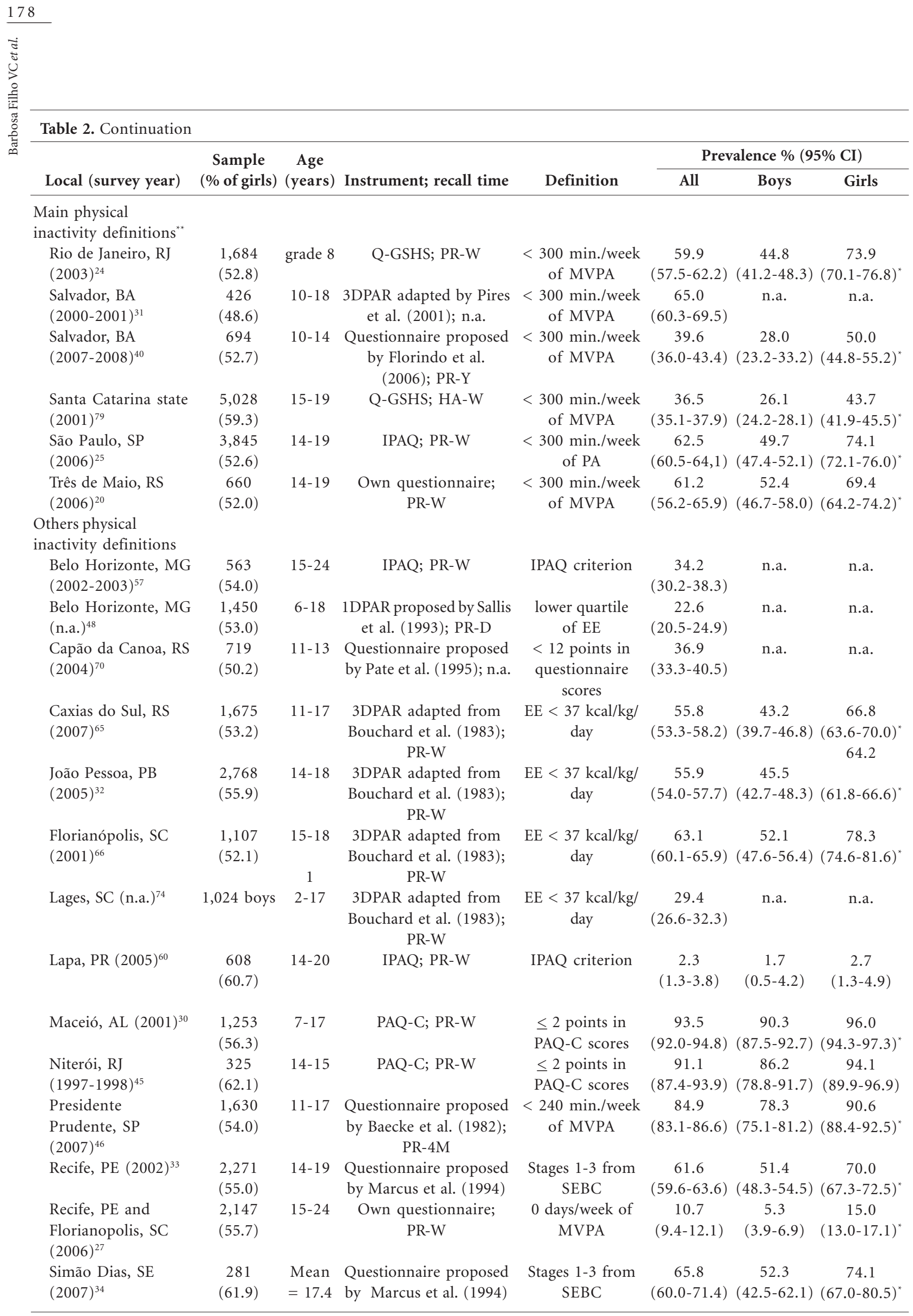


Table 2. Continuation

\begin{tabular}{|c|c|c|c|c|c|c|c|}
\hline \multirow[b]{2}{*}{ Local (survey year) } & \multirow{2}{*}{$\begin{array}{l}\text { Sample } \\
(\% \text { of girls })\end{array}$} & \multirow{2}{*}{$\begin{array}{c}\text { Age } \\
(\text { years })\end{array}$} & \multirow{2}{*}{ Instrument; recall time } & \multirow[b]{2}{*}{ Definition } & \multicolumn{3}{|c|}{ Prevalence \% (95\% CI) } \\
\hline & & & & & All & Boys & Girls \\
\hline \multicolumn{8}{|l|}{$\begin{array}{l}\text { Low Practice of PA or } \\
\text { Sports }\end{array}$} \\
\hline $\begin{array}{l}\text { Bento Gonçalves, RS } \\
\text { (n.a. })^{71}\end{array}$ & $\begin{array}{c}590 \\
(58.5)\end{array}$ & $9-18$ & $\begin{array}{c}\text { Own questionnaire; } \\
\text { HA-W }\end{array}$ & $\begin{array}{c}<3 \text { days/week of } \\
\text { PA }\end{array}$ & $\begin{array}{c}52.3 \\
(48.2-56.4)\end{array}$ & n.a. & n.a. \\
\hline $\begin{array}{l}\text { Caxias do Sul, RS } \\
(2007)^{21}\end{array}$ & $\begin{array}{l}1,442 \\
(50.0)\end{array}$ & $7-12$ & $\begin{array}{c}\text { Own questionnaire; } \\
\text { HA-W }\end{array}$ & $\begin{array}{c}\text { No weekly PA or } \\
\text { sports }\end{array}$ & $\begin{array}{c}61.3 \\
(58.7-63.9)\end{array}$ & n.a. & n.a. \\
\hline Cuiabá, MT (1998) ${ }^{42}$ & $\begin{array}{l}2,291 \\
(56.1)\end{array}$ & $10-20$ & $\begin{array}{c}\text { Own questionnaire; } \\
\text { HA-W }\end{array}$ & $\begin{array}{c}\text { No weekly PA or } \\
\text { sports }\end{array}$ & $\begin{array}{c}32.9 \\
(30.9-34.8)\end{array}$ & n.a. & n.a. \\
\hline Cuiabá, MT (2008) $)^{43}$ & $\begin{array}{l}1,209 \\
(55.4)\end{array}$ & $14-19$ & $\begin{array}{c}\text { Own questionnaire; } \\
\text { HA-W }\end{array}$ & $\begin{array}{c}\text { No weekly PA or } \\
\text { sports }\end{array}$ & $\begin{array}{c}19.1 \\
(16.9-21.4)\end{array}$ & n.a. & n.a. \\
\hline $\begin{array}{l}\text { Northeast and } \\
\text { Southeast of Brazil } \\
(1996-1997)^{28}\end{array}$ & $\begin{array}{l}1,881 \\
(49.0)\end{array}$ & $15-20$ & $\begin{array}{l}\text { Own questionnaire; } \\
\text { HA-M }\end{array}$ & $\begin{array}{c}<3 \text { days/week of } \\
\text { PA }\end{array}$ & $\begin{array}{c}79.6 \\
(77.7-81.4)\end{array}$ & $\begin{array}{c}67.7 \\
(64.7-70.7)\end{array}$ & $\begin{array}{c}92.1 \\
(90.1-93.7)^{*}\end{array}$ \\
\hline Pelotas, RS $(1998)^{81}$ & $\begin{array}{l}2,410 \\
(56.4)\end{array}$ & $10-19$ & $\begin{array}{c}\text { Own questionnaire; } \\
\text { HA-Y }\end{array}$ & $\begin{array}{c}<3 \text { days/week of } \\
\text { PA }\end{array}$ & $\begin{array}{c}29.6 \\
(27.8-31.5)\end{array}$ & n.a. & n.a. \\
\hline $\begin{array}{l}\text { Pelotas, RS } \\
(1999-2000)^{67}\end{array}$ & $\begin{array}{l}1,187 \\
(51.6)\end{array}$ & $10-19$ & Own questionnaire; n.a. & $\begin{array}{c}\text { No weekly PA or } \\
\text { sports }\end{array}$ & $\begin{array}{c}54.9 \\
(52.0-57.8)\end{array}$ & n.a. & n.a. \\
\hline $\begin{array}{l}\text { Pelotas, RS } \\
(2001-2002)^{72}\end{array}$ & $\begin{array}{c}960 \\
(51.8)\end{array}$ & $15-18$ & $\begin{array}{c}\text { Own questionnaire; } \\
\text { HA-W }\end{array}$ & $\begin{array}{c}<3 \text { days/week of } \\
\text { PA }\end{array}$ & $\begin{array}{c}39.0 \\
(35.9-42.1)\end{array}$ & $\begin{array}{c}22.2 \\
(18.5-26.3)\end{array}$ & $\begin{array}{c}54.5 \\
(50.0-59.0)^{*}\end{array}$ \\
\hline $\begin{array}{l}\text { Pelotas, RS } \\
(2005-2006)^{68}\end{array}$ & $\begin{array}{l}1,056 \\
(51.4)\end{array}$ & $11-15$ & Own questionnaire; n.a. & $\begin{array}{c}\text { No weekly PA or } \\
\text { sports }\end{array}$ & $\begin{array}{c}55.0 \\
(51.9-58.0)\end{array}$ & $\begin{array}{c}52.5 \\
(48.0-57.0)\end{array}$ & $\begin{array}{c}57.5 \\
(53.1-61.8)\end{array}$ \\
\hline Recife, PE $(2006)^{41}$ & $\begin{array}{l}1,825 \\
(60.8)\end{array}$ & $14-20$ & Q-GSHS; n.a. & $\begin{array}{c}<3 \text { days/week of } \\
\text { PA }\end{array}$ & $\begin{array}{c}42.4 \\
(40.1-44.7)\end{array}$ & n.a. & n.a. \\
\hline $\begin{array}{l}\text { Rio de Janeiro, RJ } \\
(1996)^{47}\end{array}$ & $\begin{array}{c}823 \\
(47.5)\end{array}$ & $12-20$ & $\begin{array}{c}\text { Own questionnaire; } \\
\text { HA-M }\end{array}$ & $\begin{array}{l}\text { Do not perform } \\
\text { PA or sports } \\
\text { frequently }\end{array}$ & $\begin{array}{c}75.9 \\
(72.9-78.8)\end{array}$ & $\begin{array}{c}66.7 \\
(62.0-71.1)\end{array}$ & $\begin{array}{c}86.2 \\
(82.4-89.5)^{*}\end{array}$ \\
\hline $\begin{array}{l}\text { São Leopoldo, RS } \\
(2002-2003)^{69}\end{array}$ & $\begin{array}{l}722 \\
(59.4)\end{array}$ & $10-19$ & $\begin{array}{c}\text { Questionnaire proposed } \\
\text { by Blair et al. (1985); } \\
\text { PR-W }\end{array}$ & $\begin{array}{c}\text { No weekly PA or } \\
\text { sports }\end{array}$ & $\begin{array}{c}70.0 \\
(66.5-73.3)\end{array}$ & $\begin{array}{c}55.9 \\
(49.9-61.7)\end{array}$ & $\begin{array}{c}79.6 \\
(75.4-83.3)^{*}\end{array}$ \\
\hline Santos, SP $(1997)^{58}$ & $\begin{array}{l}2,059 \\
(55.3)\end{array}$ & $13-17$ & Own questionnaire; n.a. & $\begin{array}{c}\text { No weekly PA or } \\
\text { sports }\end{array}$ & $\begin{array}{c}30.3 \\
(28.2-32.4)\end{array}$ & $\begin{array}{c}15.3 \\
(12.9-17.9)\end{array}$ & $\begin{array}{c}41.9 \\
(38.9-44.8)^{*}\end{array}$ \\
\hline $\begin{array}{l}\text { São Paulo state } \\
(2001-2002)^{49}\end{array}$ & $\begin{array}{l}1,584 \\
(50.9)\end{array}$ & $12-20$ & Own questionnaire; n.a. & $\begin{array}{c}\text { No weekly PA or } \\
\text { sports }\end{array}$ & $\begin{array}{c}28.5 \\
(26.3-30.8)\end{array}$ & n.a. & n.a. \\
\hline $\begin{array}{l}\text { Teixeira de Freitas, } \\
\text { BA }(2001)^{35}\end{array}$ & $\begin{array}{c}354 \\
(38.4)\end{array}$ & $17-19$ & Own questionnaire; n.a. & $\begin{array}{c}<3 \text { days/week of } \\
\text { PA }\end{array}$ & $\begin{array}{c}72.0 \\
(67.1-76.6)\end{array}$ & $\begin{array}{c}61.9 \\
(55.1-68.4)\end{array}$ & $\begin{array}{c}88.2 \\
(81.6-93.1)^{*}\end{array}$ \\
\hline
\end{tabular}

States of Brazil: AL: Alagoas; BA: Bahia; MG: Minas Gerais; MT: Mato Grosso; PB: Paraíba; PE: Pernambuco; PR: Paraná; RJ: Rio de Janeiro; RS: Rio Grande do Sul; SC: Santa Catarina; SE: Sergipe; SP: São Paulo. 1DPAR: 1-day physical activity record; 3DPAR: 3-day physical activity record; EE: energy expenditure; HA-W: habitual week; HA-M: habitual month; HA-Y: habitual year; IPAQ: International Physical Activity Questionnaire, short version; min./ week: minutes per week; MVPA: moderate to vigorous physical activity; n.a.: not available; PA: physical activity; PAR-Q: Physical Activity Questionnaire for Older Children; PR-D: previous day; PR-W: previous week; PR-M: previous month; PR-4M: previous 4 months; PR-Y: previous year; Q-GSHS: questionnaires based on Global School-based Student Health Survey ${ }^{13}$ and/or Youth Risk Behaviors Surveillance ${ }^{12}$ instruments; SEBC: stages of exercise behavior change. ${ }^{*}$ The $95 \%$ CIs for the prevalence of physical inactivity in boys and girls did not overlap. ${ }^{*}$ Studies considering the cutoff point used in the Global School-based Student Health Survey (less than 300 minutes/week of PA or MVPA specifically) for estimates of the prevalence of physical inactivity.

estimated a physical inactivity prevalence above $50 \%$.

Thirty-two studies $(66.7 \%)$ evaluated the prevalence of physical inactivity by gender. Only one study ${ }^{61}$ demonstrated a physical inactivity prevalence that was slightly higher among boys.
All other studies showed the highest physical inactivity prevalence among girls, with the magnitude of difference ranging from $1.0 \%{ }^{60}$ to $29.1 \%{ }^{24}$. In 28 of these studies $(87.5 \%)$, the $95 \%$ CIs for the prevalence rates in boys and girls did not overlap (Table 2). 


\section{Prevalence of sedentary behaviors}

The study characteristics and the sedentary behavior prevalence among Brazilian adolescents are shown in Table 3. Twenty-four studies presented data on the sedentary lifestyles, focusing mainly on high screen time use (13/24,
$54.2 \%)^{21,22,24,29,30,36,44,48,61,62,65,70,71}$. There were important variations in the cutoff point for determining excessive time in these activities, ranging from 2.0 to 5.5 hours per day. The cutoff of two hours daily, in turn, was the criterion most frequently used for sedentary behavior defini$\operatorname{tion}^{19,23,25,35,36,44,49,61,65,72,73}$.

Table 3. Description of the prevalence of sedentary behavior (\%) among Brazilian adolescents and its respective 95\% confidence interval (95\% CI, entire sample and by gender) of each study included in this review according to local and year of survey, sample, age, instrument, and definition of sedentary behavior.

\begin{tabular}{|c|c|c|c|c|c|c|c|}
\hline \multirow{2}{*}{ Local (survey years) } & \multirow{2}{*}{$\begin{array}{c}\text { Sample } \\
\text { (\% of girls) }\end{array}$} & \multirow{2}{*}{$\begin{array}{c}\text { Age } \\
\text { (years) }\end{array}$} & \multirow{2}{*}{ Instrument; recall time } & \multirow{2}{*}{ Definition } & \multicolumn{3}{|c|}{ Prevalence \% (95\% CI) } \\
\hline & & & & & All & Boys & Girls \\
\hline \multicolumn{8}{|l|}{ High Screen Time Use } \\
\hline $\begin{array}{l}\text { Belo Horizonte, } \\
\text { MG (n.a) }\end{array}$ & $\begin{array}{l}1,450 \\
(53.0)\end{array}$ & $6-18$ & $\begin{array}{c}\text { 1DPAR proposed by } \\
\text { Sallis et al. (1993); } \\
\text { PR-D }\end{array}$ & $>5.5$ hours/day & $\begin{array}{c}28.1 \\
(26.0-30.5)\end{array}$ & n.a. & n.a. \\
\hline $\begin{array}{l}\text { Bento Gonçalves, } \\
\text { RS (n.a.) }\end{array}$ & $\begin{array}{c}590 \\
(58.5)\end{array}$ & $9-18$ & $\begin{array}{c}\text { Own questionnaire; } \\
\text { HA-W }\end{array}$ & $5+$ hours/day & $\begin{array}{c}57.5 \\
(53.3-61.5)\end{array}$ & n.a. & n.a. \\
\hline $\begin{array}{l}\text { Capão da Canoa, } \\
\text { RS }(2004)^{70}\end{array}$ & $\begin{array}{c}719 \\
(50.2)\end{array}$ & $11-13$ & Own questionnaire; n.a. & $>4.5$ hours/day & $\begin{array}{c}31.4 \\
(28.1-35.0)\end{array}$ & n.a. & n.a. \\
\hline $\begin{array}{l}\text { Caxias do Sul, RS } \\
(2007)^{21}\end{array}$ & $\begin{array}{l}1,442 \\
(50.0)\end{array}$ & $7-12$ & $\begin{array}{c}\text { Own questionnaire; } \\
\text { HA-D }\end{array}$ & $>3$ hours/day & $\begin{array}{c}30.2 \\
(27.8-32.6)\end{array}$ & n.a. & n.a. \\
\hline $\begin{array}{l}\text { Caxias do Sul, RS } \\
(2007)^{65}\end{array}$ & $\begin{array}{l}1,675 \\
(53.2)\end{array}$ & $11-17$ & $\begin{array}{c}\text { Own questionnaire; } \\
\text { HA-D }\end{array}$ & $2+$ hours/day & $\begin{array}{c}86.6 \\
(84.9-88.3)\end{array}$ & $\begin{array}{c}89.6 \\
(87.2-91.7)\end{array}$ & $\begin{array}{c}84.0 \\
(81.3-86.4)\end{array}$ \\
\hline $\begin{array}{l}\text { Foz do Iguaçu, PR } \\
(2005)^{61}\end{array}$ & $\begin{array}{c}453 \\
(59.2)\end{array}$ & $15-18$ & $\begin{array}{c}\text { Own questionnaire; } \\
\text { PR-W }\end{array}$ & $2+$ hours/day & $\begin{array}{c}32.2 \\
(27.9-36.7)\end{array}$ & $\begin{array}{c}29.2 \\
(22.7-36.3)\end{array}$ & $\begin{array}{c}34.3 \\
(28.7-40.3)\end{array}$ \\
\hline Maceió, AL $(2001)^{30}$ & $\begin{array}{l}1,253 \\
(56.3)\end{array}$ & $7-17$ & PAQ-C; PR-W & $3+$ hours/day & $\begin{array}{c}65.0 \\
(62.3-67.7)\end{array}$ & $\begin{array}{c}63.4 \\
(59.3-67.5)\end{array}$ & $\begin{array}{c}65.3 \\
(61.7-68.9)\end{array}$ \\
\hline $\begin{array}{l}\text { Maringá, PR } \\
(2007)^{62}\end{array}$ & $\begin{array}{c}991 \\
(54.5)\end{array}$ & $14-18$ & Own questionnaire; n.a. & $4+$ hours/day & $\begin{array}{c}81.7 \\
(79.2-84.1)\end{array}$ & n.a. & n.a. \\
\hline $\begin{array}{l}\text { Ouro Preto, MG } \\
(2006)^{44}\end{array}$ & $\begin{array}{c}780 \\
(52.6)\end{array}$ & $6-14$ & Own questionnaire; n.a. & $2+$ hours/day & $\begin{array}{c}88.0 \\
(85.5-90.3)\end{array}$ & n.a. & n.a. \\
\hline \multirow[t]{2}{*}{$\begin{array}{l}\text { Pelotas, RS } \\
(2004-2005)^{22}\end{array}$} & $\begin{array}{l}4,452 \\
(49.2)\end{array}$ & $10-12$ & $\begin{array}{c}\text { Own questionnaire; } \\
\text { HA-W }\end{array}$ & $>2$ hours/day; & $\begin{array}{c}79.7 \\
(78.6-80.9)\end{array}$ & $\begin{array}{c}80.7 \\
(79.0-82.3)\end{array}$ & $\begin{array}{c}78.8 \\
(77.0-80.5)\end{array}$ \\
\hline & & & & $>4$ hours/ day & $\begin{array}{c}47.2 \\
(45.7-48.7)\end{array}$ & $\begin{array}{c}50.5 \\
(48.4-52.6)\end{array}$ & $\begin{array}{c}44.0 \\
(42.0-46.1)\end{array}$ \\
\hline $\begin{array}{l}\text { Piedade, SP } \\
(2005)^{29}\end{array}$ & $\begin{array}{c}105 \\
(63.0)\end{array}$ & $10-14$ & $\begin{array}{c}\text { Own questionnaire; } \\
\text { HA-D }\end{array}$ & $>2$ hours/day & $\begin{array}{c}73.3 \\
(63.8-81.5)\end{array}$ & n.a. & n.a. \\
\hline $\begin{array}{l}\text { Rio de Janeiro, RJ } \\
(2003)^{24}\end{array}$ & $\begin{array}{l}1,684 \\
(52.8)\end{array}$ & grade 8 & $\begin{array}{c}\text { Own questionnaire; } \\
\text { HA-W }\end{array}$ & $4+$ hours/day & $\begin{array}{c}71.7 \\
(69.5-73.9)\end{array}$ & $\begin{array}{c}71.9 \\
(68.4-75.4)\end{array}$ & $\begin{array}{c}71.5 \\
(68.6-74.4)\end{array}$ \\
\hline $\begin{array}{l}\text { São Luís, MA } \\
(2005)^{36}\end{array}$ & $\begin{array}{c}592 \\
(50.5)\end{array}$ & $9-16$ & $\begin{array}{c}\text { Own questionnaire; } \\
\text { HA-D }\end{array}$ & $2+$ hours/day & $\begin{array}{c}53.9 \\
(49.7-58.0)\end{array}$ & n.a. & n.a. \\
\hline \multicolumn{8}{|l|}{ High TV use } \\
\hline $\begin{array}{l}\text { All state capital } \\
\text { cities }(2009)^{15}\end{array}$ & $\begin{array}{c}60,973 \\
(53.3)\end{array}$ & grade 8 & Q- GSHS; HA-D & $2+$ hours/day & $\begin{array}{c}79.5 \\
(78.9-80.0)\end{array}$ & $\begin{array}{c}79.4 \\
(78.5-80.3)\end{array}$ & $\begin{array}{c}79.5 \\
(78.7-80.3)\end{array}$ \\
\hline $\begin{array}{l}\text { Curitiba, PR } \\
(2006)^{17}\end{array}$ & $\begin{array}{l}1,518 \\
(59.2)\end{array}$ & $14-18$ & $\begin{array}{c}\text { Own questionnaire; } \\
\text { HA-W }\end{array}$ & $>4$ hours/day & $\begin{array}{c}30.0 \\
(24.1-37.0)\end{array}$ & $\begin{array}{c}28.4 \\
(24.9-32.2)\end{array}$ & $\begin{array}{c}32.0 \\
(29.0-35.2)\end{array}$ \\
\hline \multirow[t]{2}{*}{ Pelotas, RS $(2003)^{73}$} & $\begin{array}{c}810 \\
(49.7)\end{array}$ & $10-19$ & Own questionnaire; n.a. & $2+$ hours/day; & $\begin{array}{c}75.4 \\
(72.3-78.4)\end{array}$ & $\begin{array}{c}73.9 \\
(69.4-78.2)\end{array}$ & $\begin{array}{c}76.9 \\
(72.5-81.0)\end{array}$ \\
\hline & & & & $4+$ hours/day & $\begin{array}{c}29.0 \\
(25.9-32.3)\end{array}$ & $\begin{array}{c}26.5 \\
(22.3-31.1)\end{array}$ & $\begin{array}{c}31.5 \\
(27.0-36.3)\end{array}$ \\
\hline $\begin{array}{l}\text { Pelotas, RS } \\
(2001-2002)^{72}\end{array}$ & $\begin{array}{c}960 \\
(51.8)\end{array}$ & $15-18$ & $\begin{array}{c}\text { Own questionnaire; } \\
\text { HA-D }\end{array}$ & $2+$ hours/day & $\begin{array}{c}74.6 \\
(71.7-77.3)\end{array}$ & n.a. & n.a. \\
\hline
\end{tabular}


Considering the 13 studies that examined the high screen time use prevalence, it was observed prevalence between $28.1 \%$ (95\% CI: $26.0-30.5)^{48}$ e $88.0 \%$ (95\% CI: 85.5-90.3 ${ }^{44}$. Eight studies $(61.5 \%)^{22,24,29,30,44,61,62,65,71}$ analyzing high screen time use and obtained an estimate above $50 \%$. In the 11 studies analyzing high TV use, the prevalence ranged from $11.3 \%$ (95\% CI: $8.1-15.1)^{35}$ to 79.5\% (95\% CI: 78.9-80.0 $)^{15}$. Six studies $(54.5 \%)^{15,23,49,69,72,73}$ obtained a high TV use prevalence above $50 \%$.

Thirteen studies (54.2\%) stratified the prevalence of sedentary behaviors by gender. Seven studies $(53.8 \%)^{15,17,30,35,46,61,73}$ identified higher sedentary behavior prevalence in girls, with the magnitude of difference ranging from $0.1 \%{ }^{15}$ to $14.8 \%{ }^{46}$. Four studies $(30.8 \%)^{19,22,24,65}$ showed a higher prevalence of sedentary behaviors among boys, with the magnitude of difference ranging from $0.4 \%^{24}$ to $6.9 \%{ }^{19}$. Two studies $(15.4 \%)^{23,69}$ found that the pattern of the gender differences change according to the cutoff point for determining the sedentary behavior outcome. Using cutoff point of two or more hours per day of TV use, boys had higher sedentary behavior; but a cutoff point of four or more hours per day resulted in a higher prevalence of girls with sedentary behavior. Only three studies $(23.1 \%)^{19,22,46}$ demonstrated no overlap between the 95\% CIs for the sedentary behavior prevalence in boys and girls (Table 3 ).

\section{Prevalence of unhealthy food habits}

The studies on unhealthy food habits are presented in Table 4. Unhealthy food habits were analyzed by 32 different articles, 24 of which $^{15,16,20,24,35,37,38,46,48-54,61,66,69,70,71,74-77}$ present-

Table 3. Continuation

\begin{tabular}{|c|c|c|c|c|c|c|c|}
\hline \multirow{2}{*}{ Local (survey years) } & \multirow{2}{*}{$\begin{array}{c}\text { Sample } \\
(\% \text { of girls })\end{array}$} & \multirow{2}{*}{$\begin{array}{c}\text { Age } \\
\text { (years) }\end{array}$} & \multirow{2}{*}{ Instrument; recall time } & \multirow{2}{*}{ Definition } & \multicolumn{3}{|c|}{ Prevalence \% (95\% CI) } \\
\hline & & & & & All & Boys & Girls \\
\hline \multicolumn{8}{|l|}{ High TV use } \\
\hline \multirow{3}{*}{$\begin{array}{l}\text { Pernambuco state } \\
(2006)^{19,16}\end{array}$} & \multirow{3}{*}{$\begin{array}{l}4,210 \\
(59.8)\end{array}$} & \multirow[t]{3}{*}{$14-20$} & \multirow[t]{3}{*}{ Q- GSHS; HA-D } & \multirow[t]{2}{*}{$2+$ hours/day; } & 40.9 & 54.0 & 47.1 \\
\hline & & & & & $(39.4-42.4)$ & $(51.6-56.4)$ & $(45.1-49.1)$ \\
\hline & & & & $3+$ hours/day & $\begin{array}{c}40.8 \\
(39.3-42.3)\end{array}$ & n.a. & n.a. \\
\hline Presidente & 1,630 & \multirow[t]{2}{*}{$11-17$} & \multirow{2}{*}{$\begin{array}{c}\text { Questionnaire proposed } \\
\text { by Baecke et al. (1982); } \\
\text { PR-4M }\end{array}$} & \multirow[t]{2}{*}{ TV use always/ } & 37.2 & 29.2 & 44.0 \\
\hline $\begin{array}{l}\text { Prudente, SP } \\
(2007)^{46}\end{array}$ & $(54.0)$ & & & & $(34.8-39.6)$ & $(26.0-32.6)$ & $(40.7-47.3)$ \\
\hline \multirow[t]{2}{*}{$\begin{array}{l}\text { Santa Catarina state } \\
(2001)^{23,83}\end{array}$} & $\begin{array}{l}5,028 \\
(59.3)\end{array}$ & \multirow[t]{2}{*}{$15-19$} & \multirow[t]{2}{*}{$\begin{array}{c}\text { Own questionnaire; } \\
\text { HA-W }\end{array}$} & $2+$ hours/day; & $\begin{array}{c}72.7 \\
(71.5-74.0)\end{array}$ & $\begin{array}{c}73.8 \\
(71.8-75.8)\end{array}$ & $\begin{array}{c}72.0 \\
(70.3-73.7)\end{array}$ \\
\hline & & & & $4+$ hours/day & $\begin{array}{c}38.5 \\
(36.7-40,3)\end{array}$ & $\begin{array}{c}37.7 \\
(35.1-40.3)\end{array}$ & $\begin{array}{c}39.1 \\
(36.8-41.5)\end{array}$ \\
\hline $\begin{array}{l}\text { São Paulo, SP } \\
(2006)^{25}\end{array}$ & $\begin{array}{l}3,845 \\
(52.6)\end{array}$ & $14-19$ & $\begin{array}{c}\text { Own questionnaire; } \\
\text { HA-D }\end{array}$ & $2+$ hours/day & $\begin{array}{c}29.1 \\
(27.7-30.6)\end{array}$ & n.a. & n.a. \\
\hline \multirow[t]{2}{*}{$\begin{array}{l}\text { São Paulo state } \\
(2001-2002)^{49}\end{array}$} & $\begin{array}{l}1,584 \\
(50.9)\end{array}$ & \multirow[t]{2}{*}{$12-20$} & \multirow[t]{2}{*}{ Own questionnaire; n.a. } & $2+$ hours/day; & $\begin{array}{c}73.9 \\
(71.7-76.1)\end{array}$ & n.a. & n.a. \\
\hline & & & & $>4$ hours/day & $\begin{array}{c}17.6 \\
(15.8-19.6)\end{array}$ & n.a. & n.a. \\
\hline \multirow[t]{2}{*}{$\begin{array}{l}\text { São Leopoldo, RS } \\
(2002-2003)^{69,82}\end{array}$} & $\begin{array}{c}722 \\
(59.4)\end{array}$ & \multirow[t]{2}{*}{$10-19$} & \multirow{2}{*}{$\begin{array}{c}\text { Questionnaire proposed } \\
\text { by Blair et al. (1985); } \\
\text { PR-W }\end{array}$} & $>2$ hours/day; & $\begin{array}{c}75.1 \\
(71.7-78.2)\end{array}$ & $\begin{array}{c}75.7 \\
(70.3-80.5)\end{array}$ & $\begin{array}{c}74.6 \\
(70.2-78.7)\end{array}$ \\
\hline & & & & $>4$ hours/day & $\begin{array}{c}41.0 \\
(37.4-44.7)\end{array}$ & $\begin{array}{c}40.3 \\
(34.6-46.1)\end{array}$ & $\begin{array}{c}41.7 \\
(37.0-46.5)\end{array}$ \\
\hline \multirow[t]{2}{*}{$\begin{array}{l}\text { Teixeira de Freitas, } \\
\text { BA }(2001)^{35}\end{array}$} & $\begin{array}{c}354 \\
(38.4)\end{array}$ & \multirow[t]{2}{*}{$17-19$} & \multirow[t]{2}{*}{ Own questionnaire; n.a. } & $2+$ hours/day; & $\begin{array}{c}47.5 \\
(42.0-53.0)\end{array}$ & $\begin{array}{c}43.7 \\
(36.8-50.8)\end{array}$ & $\begin{array}{c}53.4 \\
(44.5-62.2)\end{array}$ \\
\hline & & & & $>4$ hours/day & $\begin{array}{c}11.3 \\
(8.1-15.1)\end{array}$ & $\begin{array}{c}10.7 \\
(6.8-15.7)\end{array}$ & $\begin{array}{c}12.2 \\
(7.1-19.1)\end{array}$ \\
\hline
\end{tabular}

States of Brazil: AL: Alagoas; BA: Bahia; MA: Maranhão; MG: Minas Gerais; PR: Paraná; RJ: Rio de Janeiro; RS: Rio Grande do Sul; SP: São Paulo. 1DPAR: 1-day physical activity record; HA-D: habitual day; HA-W: habitual week; n.a.: not available; PAR-Q: Physical Activity Questionnaire for Older Children; PR-D: previous day; PR-W: previous week; PR-4M: previous 4 months; Q-GSHS: questionnaires based on Global School-based Student Health Survey ${ }^{13}$ and/or Youth Risk Behaviors Surveillance ${ }^{12}$ instruments. ${ }^{*}$ The $95 \%$ CIs for the prevalence of physical inactivity in boys and girls did not overlap. 
ed outcomes related to high consumption of unhealthy foods. Ten studies (31.2\%) examined the prevalence of unhealthy food habits based on the high consumption of fat-rich foods ${ }^{48,51,70,75}$ or by analyzing the daily energy intake and classifying it according to national and international recommendations for a healthy $\operatorname{diet}^{20,37,38,49,54,69}$. In these studies, the prevalence of unhealthy food habits ranged from $30.1 \%$ (95\% CI: 26.4-34.1) ${ }^{38}$ to $98.3 \%(95 \% \text { CI: } 97.0-99.2)^{20}$. Six of these studies $(60 \%)^{20,37,48,49,54,69}$ estimated an unhealthy food habit prevalence above $50 \%$.

Other studies evaluated the consumption of unhealthy foods (e.g., soft drinks or sweets). High soft drink consumption was the most studied outcome $(37.5 \%)^{15,16,24,35,48,50,52,61,71,74,76,77}$, followed by the high sweets consumption $(31.2 \%)^{15}$, $24,48,50,54,66,74,71,76,77$. Overall, the prevalence of high soft drink consumption varied from 20.4\% (95\% CI: $18.9-22.0)^{77}$ to $71.0 \%$ (95\% CI: $\left.67.2-74.6\right)^{71}$. Most studies that examined the high soft drink consumption $(84.6 \%)^{15,16,24,35,38,48,50,52,61,74,71}$ have found a prevalence rate above $30 \%$. In the 10 studies analyzing the high sweets consumption, the prevalence ranged from $20.1 \%$ (95\% CI: 17.8 $22.5)^{66}$ to $96.9 \%$ (95\% CI: $\left.94.7-98.4\right)^{54}$. Four of these studies ( $40 \%)^{15,48,54,77}$ estimated a prevalence of high sweets consumption above $50 \%$. Others unhealthy foods were analyzed in a few studies included in this review (e.g., fast or fried foods). Thus, they were only presented in the Table 4 .

A total of 23 studies $(71.9 \%)^{15,16,24,26,31,32,46,48 \text {, }}$ 50,54,55,59,61,66,69,71,74-80,82 analyzed low healthy food consumption outcomes. Nine studies $(28.1 \%)^{31,48,}$ $55,59,69,75,79,80,82$ estimated this factor based on dietary fiber intake or fiber-rich foods intake (fruits and vegetables specifically or in a clustering with other fiber-rich foods). These studies have estimated the low healthy food consumption prevalence ranged from $46.5 \%$ (95\% CI: 45.1-47.9) ${ }^{79}$ to $94.7 \%$ (95\% CI: $93.7-95.6)^{80}$. Seven of these studies $(77.8 \%)^{31,55,59,69,75,80,82}$ estimated a prevalence rate above $50 \%$.

Thirteen studies $(40.6 \%)^{15,16,24,32,46,50,54,61 \text {, }}$ $66,71,74,76,78$ specifically analyzed the low fruit consumption, with the prevalence ranging from $33.4 \%$ (95\% CI: $31.9-34.8)^{16}$ to $82.8 \%$ (95\% CI: $79.0-86.1)^{61}$. Nine of these studies $(69.2 \%)^{15,24,46 \text {, }}$ $50,54,61,66,76,78$ estimated a prevalence rate above $50 \%$. Likewise, 11 studies $(34.4 \%)^{16,26,32,46,54,61 \text {, }}$ $66,71,74,76,78$ found prevalence of low vegetable consumption ranging from $36.3 \%$ (95\% CI: 34.9 $37.8)^{16}$ to $75.8 \%$ (95\% CI: 73.7-77.9 $)^{46}$. Six of these studies $(54.5 \%)^{26,32,46,54,61,66}$ observed a prevalence rate above $50 \%$.
Only 10 articles (31.2\%) showed results stratified by gender on high unhealthy food consumption, leading to the evaluation of 13 outcomes (e.g., high consumption of sweet, fast food, soft drinks and others). Of these outcomes, seven $(53.8 \%)^{15,20,24,52,61,66,75}$ presented higher estimates among boys, with the magnitude of difference ranging from $0.4 \%{ }^{75}$ to $4.3 \% \%^{52}$. Six outcomes $(46.2 \%)^{15,24,35,46,66,69}$ were higher among girls, with the magnitude of difference ranging from $2.7 \%{ }^{66}$ to $18.6 \%{ }^{24}$. However, these gender differences were discrete in most outcomes; only two studies $^{15,24}$ obtained no overlap between the $95 \%$ CIs for the prevalence in boys and girls (Table 4).

Eleven studies (34.4\%) evaluating the prevalence of low healthy food consumption stratified their results by gender, leading to the evaluating of 17 outcomes. Of these outcomes, eleven $(64.7 \%)^{15,26,32,46,66,75,78-80}$ showed a higher prevalence among boys, with the magnitude of difference ranging from $0.1 \%^{26,46}$ to $10.1 \%{ }^{79}$. Five outcomes $(29.4 \%)^{24,61,69,78}$ were higher among girls and the magnitude of difference ranged from $2.7 \%^{24}$ to $19.3 \% \%^{69}$. One study $y^{46}$ found a similar prevalence between genders for the low consumption of fruits. The magnitude of these differences was also discrete; only three studies ${ }^{66,69,79} \mathrm{ob}-$ tained no overlap between the $95 \%$ CIs for the prevalence in boys and girls (Table 4).

\section{Discussion}

\section{Study characteristics}

The studies included in this review have some characteristics that should be highlighted. First, the examined studies indicated a growing interest in epidemiological research related to physical activity/inactivity among adolescents (70\% of studies assessed this behavior). This evolution of physical activity-related literature in Brazil has also been observed in previous reviews ${ }^{84,85}$. This review also identified a large number of studies with survey periods between 2005 and 2009, indicating the growing national scientific interest in the assessment of behaviors related to physical activity, a sedentary lifestyle, and healthy food habits.

The scientific interest in evaluating these behavioral risk factors among Brazilian youth also has an impact on aspects related to the territorial coverage of the studies. Some included studies were conducted with adolescents from a Brazilian state specifically (e.g., Pernambuco and São Paulo states) ${ }^{16,19,49,79}$ or from two or more re- 
Table 4. Description of prevalence of unhealthy food habits (\%) among Brazilian adolescents and its respective 95\% confidence interval (95\% CI, entire sample and by gender) of each study included in this review according to local and year of survey, sample, age, instrument, and definition of unhealthy food habit.

\begin{tabular}{|c|c|c|c|c|c|c|c|}
\hline \multirow{2}{*}{$\begin{array}{c}\text { Local } \\
\text { (survey years) }^{* *}\end{array}$} & \multirow{2}{*}{$\begin{array}{c}\text { Sample } \\
\text { (\% of girls) }\end{array}$} & \multirow{2}{*}{$\begin{array}{c}\text { Age } \\
\text { (years) }\end{array}$} & \multirow{2}{*}{ Instrument; recall time } & \multirow[b]{2}{*}{ Definition } & \multicolumn{3}{|c|}{ Prevalence \% (95\% CI) } \\
\hline & & & & & All & Boys & Girls \\
\hline \multicolumn{8}{|l|}{$\begin{array}{l}\text { High unhealthy food } \\
\text { consumption }\end{array}$} \\
\hline \multirow[t]{2}{*}{$\begin{array}{l}\text { All state capital } \\
\text { cities }(2009)^{15}\end{array}$} & $\begin{array}{c}60,973 \\
(53.3)\end{array}$ & grade 8 & FFQ-GSHS; PR-W & $\begin{array}{l}\text { Soft drinks } 5+ \\
\text { days/week; }\end{array}$ & $\begin{array}{c}37.2 \\
(36.5-37.9)\end{array}$ & $\begin{array}{c}37.9 \\
(36.9-38.9)\end{array}$ & $\begin{array}{c}36.6 \\
(35.7-37.5)\end{array}$ \\
\hline & & & & $\begin{array}{l}\text { Sweets } 5+\text { days/ } \\
\text { week }\end{array}$ & $\begin{array}{c}50.9 \\
(50.1-51.6)\end{array}$ & $\begin{array}{c}42.6 \\
(41.5-43.6)\end{array}$ & $\begin{array}{c}58.3 \\
(57.4-59.3)^{*}\end{array}$ \\
\hline \multirow[t]{2}{*}{ Bauru, SP (n.a.) ${ }^{50}$} & $\begin{array}{c}414 \\
\text { (n.a.) }\end{array}$ & $12-16$ & FFQ-14 items; n.a. & $\begin{array}{l}\text { Soft drinks } 1+ \\
\text { times/day; }\end{array}$ & $\begin{array}{c}43.3 \\
(38.5-48.3)\end{array}$ & n.a. & n.a. \\
\hline & & & & $\begin{array}{c}\text { Sweets } 1+\text { times/ } \\
\text { day }\end{array}$ & $\begin{array}{c}35.3 \\
(30.7-40.1)\end{array}$ & n.a. & n.a. \\
\hline \multirow[t]{3}{*}{$\begin{array}{l}\text { Belo Horizonte, } \\
\text { MG (n.a.) }{ }^{48}\end{array}$} & $\begin{array}{l}1,450 \\
(53.0)\end{array}$ & $6-18$ & $\begin{array}{l}\text { FFQ proposed by Block } \\
\text { et al. (2000); PR-Y }\end{array}$ & $\begin{array}{c}\text { Soft drinks 6+ } \\
\text { days/week; }\end{array}$ & $\begin{array}{c}32.9 \\
(30.4-35.4)\end{array}$ & n.a. & n.a. \\
\hline & & & & $\begin{array}{c}\text { Sweets } 6+\text { days/ } \\
\text { week; }\end{array}$ & $\begin{array}{c}58.3 \\
(55.7-60.8)\end{array}$ & n.a. & n.a. \\
\hline & & & & $\begin{array}{l}\text { Very high fat } \\
\text { content and very } \\
\text { low FVC in } \\
\text { habitual food } \\
\text { intake }\end{array}$ & $\begin{array}{c}64.8 \\
(62.2-67.2)\end{array}$ & n.a. & n.a. \\
\hline \multirow[t]{4}{*}{$\begin{array}{l}\text { Bento Gonçalves, } \\
\text { RS (n.a })^{71}\end{array}$} & $\begin{array}{c}590 \\
(58.5)\end{array}$ & $9-18$ & FFQ; n.a. & $\begin{array}{c}\text { Animal fats } 4+ \\
\text { days/week; }\end{array}$ & $\begin{array}{c}24.4 \\
(21.0-28.1)\end{array}$ & n.a. & n.a. \\
\hline & & & & $\begin{array}{l}\text { Fast foods 4+ } \\
\text { days/week; }\end{array}$ & $\begin{array}{c}70.3 \\
(66.4-73.9)\end{array}$ & n.a. & n.a. \\
\hline & & & & $\begin{array}{l}\text { Soft drinks } 4+ \\
\text { days/week; }\end{array}$ & $\begin{array}{c}71.0 \\
(67.2-74.6)\end{array}$ & n.a. & n.a. \\
\hline & & & & $\begin{array}{c}\text { Sweets } 4+\text { days/ } \\
\text { week }\end{array}$ & $\begin{array}{c}42.7 \\
(38.7-46.8)\end{array}$ & n.a. & n.a. \\
\hline $\begin{array}{l}\text { Capão da Canoa, } \\
\text { RS }(2004)^{70}\end{array}$ & $\begin{array}{c}719 \\
(50.2)\end{array}$ & $11-13$ & $\begin{array}{l}\text { FFQ proposed by Chiara } \\
\text { \& Sichieri (2001); PR-M }\end{array}$ & $\begin{array}{l}120+\text { points } \\
\text { estimated by a } \\
\text { list of } 9 \text { high- } \\
\text { energy density } \\
\text { foods }\end{array}$ & $\begin{array}{c}50.1 \\
(46.3-53.8)\end{array}$ & n.a. & n.a. \\
\hline \multirow[t]{2}{*}{$\begin{array}{l}\text { Florianópolis, SC } \\
(2001)^{66}\end{array}$} & $\begin{array}{l}1,107 \\
(52.1)\end{array}$ & $15-18$ & FFQ-GSHS; PR-W & $\begin{array}{l}\text { Fried Foods 1+ } \\
\text { times/day; }\end{array}$ & $\begin{array}{c}7.7 \\
(6.3-9.2)\end{array}$ & $\begin{array}{c}8.9 \\
(6.6-11.6)\end{array}$ & $\begin{array}{c}6.9 \\
(5.3-8.8)\end{array}$ \\
\hline & & & & $\begin{array}{c}\text { Sweets } 1+\text { times/ } \\
\text { day }\end{array}$ & $\begin{array}{c}20.1 \\
(17.8-22.5)\end{array}$ & $\begin{array}{c}18.8 \\
(15.6-22.3)\end{array}$ & $\begin{array}{c}21.5 \\
(18.3-25.0)\end{array}$ \\
\hline $\begin{array}{l}\text { Foz do Iguacu, PR } \\
(2005)^{61}\end{array}$ & $\begin{array}{c}453 \\
(59.2)\end{array}$ & $15-18$ & FFQ-GSHS; PR-W & $\begin{array}{l}\text { Soft drinks } 1+ \\
\text { times/day }\end{array}$ & $\begin{array}{c}42.8 \\
(38.2-47.5)\end{array}$ & $\begin{array}{c}43.8 \\
(36.5-51.2)\end{array}$ & $\begin{array}{c}42.2 \\
(36.2-48.3)\end{array}$ \\
\hline \multirow[t]{3}{*}{ Lages, SC (n.a.) $)^{74}$} & 1,024 boys & $12-17$ & FFQ-GSHS; PR-W & $\begin{array}{l}\text { Fried Foods 4+ } \\
\text { days/week; }\end{array}$ & $\begin{array}{c}29.1 \\
(26.3-32.0)\end{array}$ & n.a. & n.a. \\
\hline & & & & $\begin{array}{l}\text { Soft Drinks 4+ } \\
\text { days/week; }\end{array}$ & $\begin{array}{c}36.8 \\
(33.8-39.8)\end{array}$ & n.a. & n.a. \\
\hline & & & & $\begin{array}{c}\text { Sweets } 4+\text { days/ } \\
\text { week }\end{array}$ & $\begin{array}{c}39.6 \\
(36.6-42.7)\end{array}$ & n.a. & n.a. \\
\hline Niterói, RJ $(2003)^{51}$ & $\begin{array}{c}539 \\
(63.4)\end{array}$ & $12-19$ & $\begin{array}{l}\text { FFQ proposed by Chiara } \\
\text { \& Sichieri (2001); PR-M }\end{array}$ & $\begin{array}{l}\text { 120+ points } \\
\text { estimated by a } \\
\text { list of } 9 \text { high- } \\
\text { energy density } \\
\text { foods }\end{array}$ & $\begin{array}{c}38.0 \\
(33.9-42.3)\end{array}$ & n.a. & n.a. \\
\hline
\end{tabular}




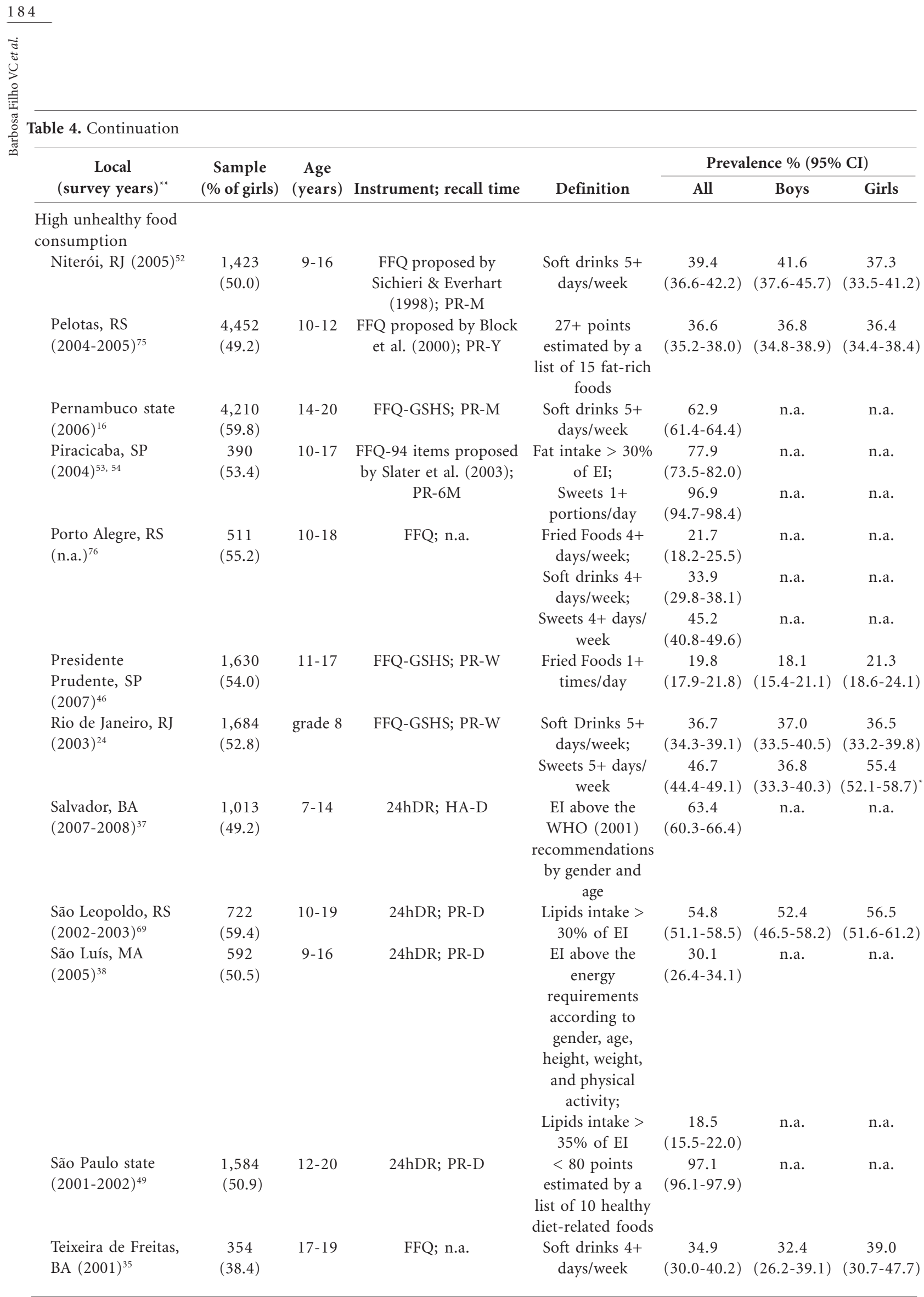


Table 4. Continuation

\begin{tabular}{|c|c|c|c|c|c|c|c|}
\hline \multirow{2}{*}{$\begin{array}{c}\text { Local } \\
\text { (survey years) }^{* *}\end{array}$} & \multirow{2}{*}{$\begin{array}{c}\text { Sample } \\
\text { (\% of girls) }\end{array}$} & \multirow{2}{*}{$\begin{array}{c}\text { Age } \\
\text { (years) }\end{array}$} & \multirow{2}{*}{ Instrument; recall time } & \multirow[b]{2}{*}{ Definition } & \multicolumn{3}{|c|}{ Prevalence \% (95\% CI) } \\
\hline & & & & & All & Boys & Girls \\
\hline \multicolumn{8}{|l|}{$\begin{array}{l}\text { High unhealthy food } \\
\text { consumption }\end{array}$} \\
\hline \multirow[t]{2}{*}{$\begin{array}{l}\text { Toledo, PR } \\
(2003)^{77}\end{array}$} & \multirow[t]{2}{*}{$\begin{array}{l}2,562 \\
(55.5)\end{array}$} & \multirow[t]{2}{*}{$14-19$} & \multirow{2}{*}{$\begin{array}{l}\text { FFQ proposed by } \\
\text { Sichieri \& Everhart } \\
\text { (1998); PR-M }\end{array}$} & $\begin{array}{l}\text { Soft drinks } 1+ \\
\text { times/day; }\end{array}$ & $\begin{array}{c}20.4 \\
(18.9-22.0)\end{array}$ & n.a. & n.a. \\
\hline & & & & $\begin{array}{c}\text { Sweets } 1+\text { times/ } \\
\text { day }\end{array}$ & $\begin{array}{c}59.5 \\
(57.6-61.4)\end{array}$ & n.a. & n.a. \\
\hline $\begin{array}{l}\text { Três de Maio, RS } \\
(2006)^{20}\end{array}$ & $\begin{array}{c}660 \\
(52.0)\end{array}$ & $14-19$ & FFQ; HA-D & $\begin{array}{l}\text { At least one } \\
\text { component out } \\
\text { of the daily } \\
\text { intake } \\
\text { recommendations } \\
\text { (SBC, 2005) }\end{array}$ & $\begin{array}{c}98.3 \\
(97.0-99.2)\end{array}$ & $\begin{array}{c}99.4 \\
(97.7-99.9)\end{array}$ & $\begin{array}{c}97.4 \\
(95.1-98.8)\end{array}$ \\
\hline \multicolumn{8}{|l|}{$\begin{array}{l}\text { Low healthy food } \\
\text { consumption }\end{array}$} \\
\hline \multirow[t]{2}{*}{$\begin{array}{l}\text { All state capital } \\
\text { cities }(2009)^{15,26}\end{array}$} & \multirow[t]{2}{*}{$\begin{array}{c}60,973 \\
(53.3)\end{array}$} & \multirow[t]{2}{*}{ grade 8} & \multirow[t]{2}{*}{ FFQ-GSHS; PR-W } & $\mathrm{FC}<5$ days/week; & $\begin{array}{c}69.5 \\
(67.8-69.2)\end{array}$ & $\begin{array}{c}69.6 \\
(67.6-69.6)\end{array}$ & $\begin{array}{c}69.4 \\
(67.5-69.3)\end{array}$ \\
\hline & & & & $\mathrm{VC}<5$ days/week & $\begin{array}{c}69.8 \\
(69.4-70.2)\end{array}$ & $\begin{array}{c}69.8 \\
(69.3-70.3)\end{array}$ & $\begin{array}{c}69.7 \\
(69.2-70.2)\end{array}$ \\
\hline $\begin{array}{l}\text { Bauru, SP } \\
(\text { n.a. })^{50}\end{array}$ & $\begin{array}{l}414 \\
\text { (n.a.) }\end{array}$ & $12-16$ & FFQ-14 items; n.a. & $\mathrm{FC}<1$ time/day & $\begin{array}{c}69.6 \\
(64.9-74.0)\end{array}$ & n.a. & n.a. \\
\hline $\begin{array}{l}\text { Belo Horizonte, } \\
\text { MG (n.a.) }\end{array}$ & $\begin{array}{l}1,450 \\
(53.0)\end{array}$ & $6-18$ & $\begin{array}{l}\text { FFQ proposed by Block } \\
\text { et al. }(2000) ; \text { PR-Y }\end{array}$ & $\begin{array}{c}\text { FVC }<1 \text { times } / \\
\text { day }\end{array}$ & $\begin{array}{c}52.0 \\
(49.4-54.6)\end{array}$ & n.a. & n.a. \\
\hline \multirow[t]{2}{*}{$\begin{array}{l}\text { Bento Gonçalves, } \\
\text { RS (n.a) })^{71}\end{array}$} & \multirow[t]{2}{*}{$\begin{array}{c}590 \\
(58.5)\end{array}$} & \multirow[t]{2}{*}{$9-18$} & \multirow[t]{2}{*}{ FFQ; n.a. } & $\mathrm{FC}<4$ days/week; & $\begin{array}{c}36.8 \\
(32.8-40.8)\end{array}$ & n.a. & n.a. \\
\hline & & & & $\begin{array}{c}\text { Green } \mathrm{VC}<4 \\
\text { days/week }\end{array}$ & $\begin{array}{c}49.5 \\
(45.4-53.6)\end{array}$ & n.a. & n.a. \\
\hline \multirow[t]{2}{*}{$\begin{array}{l}\text { Florianópolis, SC } \\
(2001)^{66}\end{array}$} & \multirow[t]{2}{*}{$\begin{array}{l}1,107 \\
(52.1)\end{array}$} & \multirow[t]{2}{*}{$15-18$} & \multirow[t]{2}{*}{ FFQ-GSHS; PR-W } & $\mathrm{FC}<1$ time/day; & $\begin{array}{c}66.9 \\
(64.0-69.7)\end{array}$ & $\begin{array}{c}69.7 \\
(65.5-73.6)\end{array}$ & $\begin{array}{c}64.3 \\
(60.2-68.3)\end{array}$ \\
\hline & & & & $\mathrm{VC}<1$ time/day & $\begin{array}{c}69.6 \\
(67.0-72.1)\end{array}$ & $\begin{array}{c}74.3 \\
(71.0-77.9)\end{array}$ & $\begin{array}{c}65.8 \\
(62.2-69.3)\end{array}$ \\
\hline \multirow[t]{2}{*}{$\begin{array}{l}\text { Foz do Iguacu, PR } \\
(2005)^{61}\end{array}$} & \multirow[t]{2}{*}{$\begin{array}{c}453 \\
(59.2)\end{array}$} & \multirow[t]{2}{*}{$15-18$} & \multirow[t]{2}{*}{ FFQ-GSHS; PR-W } & $\mathrm{FC}<1$ day & $\begin{array}{c}82.8 \\
(79.0-86.1)\end{array}$ & $\begin{array}{c}81.1 \\
(74.7-86.4)\end{array}$ & $\begin{array}{c}84.0 \\
(79.0-88.1)\end{array}$ \\
\hline & & & & $\mathrm{VC}<1$ day & $\begin{array}{c}73.7 \\
(69.4-77.7)\end{array}$ & $\begin{array}{c}69.7 \\
(62.6-76.2)\end{array}$ & $\begin{array}{c}76.5 \\
(70.9-81.4)\end{array}$ \\
\hline \multirow[t]{2}{*}{$\begin{array}{l}\text { João Pessoa, PB } \\
(2005)^{32}\end{array}$} & $\begin{array}{l}2,768 \\
(55.9)\end{array}$ & $14-18$ & FFQ-GSHS; HA-W & FC $<5$ days/week; & $\begin{array}{c}48.2 \\
(46.3-50.0)\end{array}$ & $\begin{array}{c}49.1 \\
(46.3-51.9)\end{array}$ & $\begin{array}{c}47.5 \\
(45.0-50.0)\end{array}$ \\
\hline & & & & $\mathrm{VC}<5$ days/week & $\begin{array}{c}57.8 \\
(53.8-57.6)\end{array}$ & $\begin{array}{c}60.4 \\
(57.6-63.1)\end{array}$ & $\begin{array}{c}55.7 \\
(53.2-58.2)\end{array}$ \\
\hline Lages, SC (n.a.) ${ }^{74}$ & 1,024 boys & $12-17$ & FFQ-GSHS; PR-W & FC $<4$ days/week; & $\begin{array}{c}38.9 \\
(35.9-41.9)\end{array}$ & n.a. & n.a. \\
\hline Londrina, PR & 664 & $15-18$ & FFQ-GSHS; PR-W & $\mathrm{VC}<4$ days/week & $\begin{array}{c}51.5 \\
(48.3-54.6)\end{array}$ & n.a. & n.a. \\
\hline$(2005)^{78}$ & $(61.6)$ & & & $\mathrm{FC}<4$ days/week; & $\begin{array}{c}56.7 \\
(52.7-60.5)\end{array}$ & $\begin{array}{c}53.0 \\
(46.6-59.4)\end{array}$ & $\begin{array}{c}58.9 \\
(53.9-63.8)\end{array}$ \\
\hline & & & & $\mathrm{VC}<4$ days/week & $\begin{array}{c}43.9 \\
(40.0-47.9)\end{array}$ & $\begin{array}{c}48.2 \\
(41.8-54.6)\end{array}$ & $\begin{array}{c}41.3 \\
(36.4-46.3)\end{array}$ \\
\hline Pelotas, RS $(2004)^{80}$ & $\begin{array}{l}2,209 \\
(55.6)\end{array}$ & $13-14$ & $\begin{array}{l}\text { FFQ proposed by Block } \\
\text { et al. }(2000) \text {; PR-Y }\end{array}$ & $\begin{array}{c}\text { FVC }<5 \text { times } / \\
\text { day }\end{array}$ & $\begin{array}{c}94.7 \\
(93.7-95.6)\end{array}$ & $\begin{array}{c}95.1 \\
(93.5-96.4)\end{array}$ & $\begin{array}{c}94.3 \\
(92.8-95.5)\end{array}$ \\
\hline $\begin{array}{l}\text { Pelotas, RS } \\
(2004-2005)^{75}\end{array}$ & $\begin{array}{l}4,452 \\
(49.2)\end{array}$ & $10-12$ & $\begin{array}{l}\text { FFQ proposed by Block } \\
\text { et al. }(2000) \text {; PR-Y }\end{array}$ & $\begin{array}{c}<20 \text { points } \\
\text { estimated by a } \\
\text { list of } 9 \text { fiber-rich } \\
\text { foods }\end{array}$ & $\begin{array}{c}83.9 \\
(82.7-84.9)\end{array}$ & $\begin{array}{c}84.4 \\
(82.8-85.9)\end{array}$ & $\begin{array}{c}83.5 \\
(81.9-85.0)\end{array}$ \\
\hline
\end{tabular}




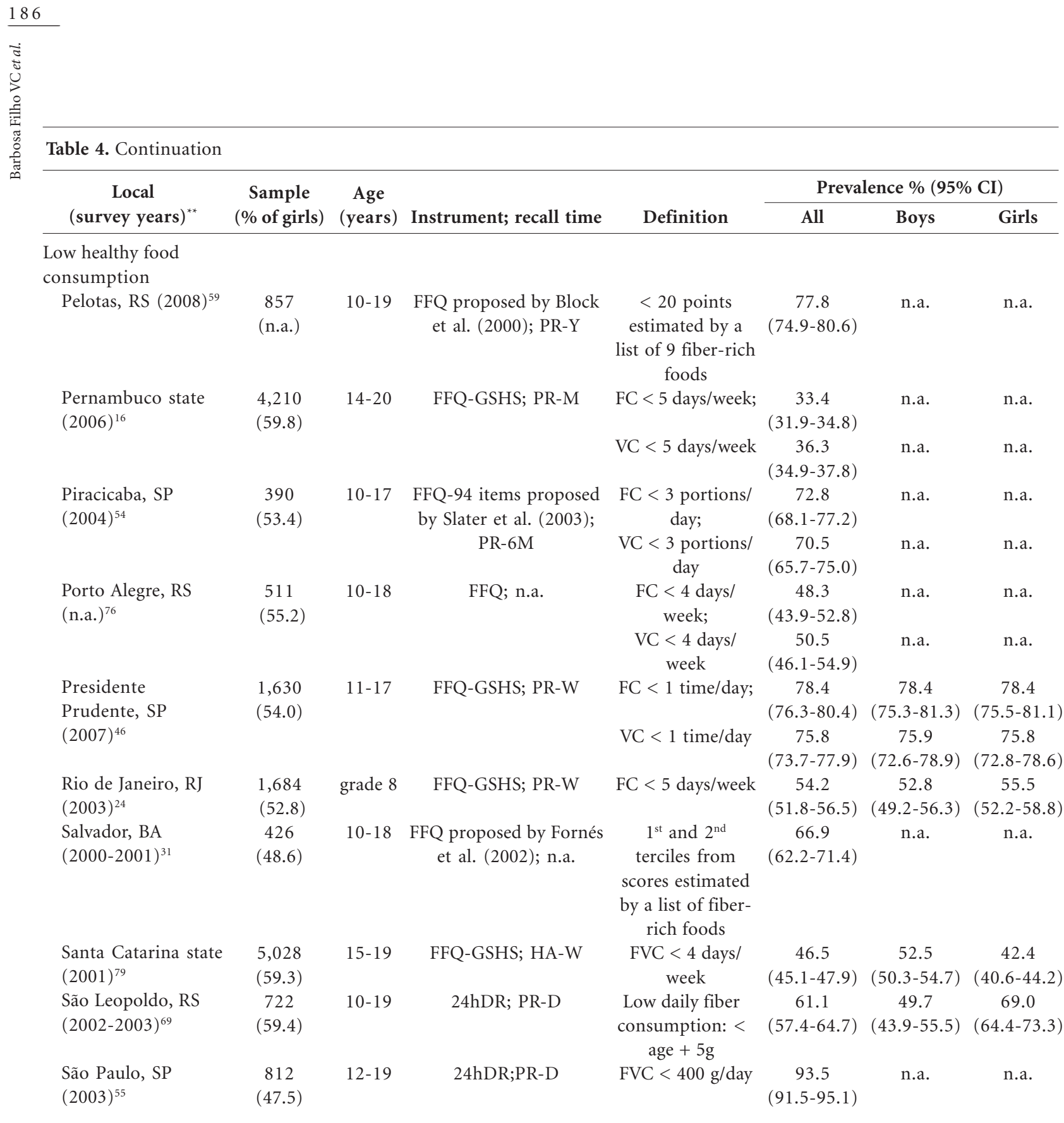

States of Brazil: BA: Bahia; MA: Maranhão; MG: Minas Gerais; PB: Paraíba; PR: Paraná; RJ: Rio de Janeiro; RS: Rio Grande do Sul; SC: Santa Catarina; SP: São Paulo; 24hDR: 24-hour dietary recall; EI: energy intake; FC: fruit consumption; FFQ-GSHS: food frequency questionnaires based on Global Schoolbased Student Health Survey ${ }^{13}$ and/or Youth Risk Behaviors Surveillance ${ }^{12}$ instruments; FVC: fruit and vegetable consumption; HA-D: habitual day; HA-W: habitual week; n.a.: not available; PR-D: previous day; PR-W: previous week; PR-M: previous month; PR-6M: previous 6 months; PR-Y: previous year; SBC: Sociedade Brasileira de Cardiologia; VC: vegetable consumption; WHO: World Health Organization. ${ }^{\star}$ The $95 \%$ CIs for the prevalence of physical inactivity in boys and girls did not overlap. ${ }^{*}$ Some studies appear once or more than in the table because they used different cutoff points for food habits.

gions in Brazil ${ }^{15,26-28}$. These studies contributed to the research comprehensiveness to unexplored territories of Brazil, including small cities and poorer regions of a state. Additionally, several studies adopted a comprehensive sample selection procedure, based on the youth population (household surveys) or they were school-based surveys considering both public and private schools (see Table 1).

Two other characteristics, however, indicated that there are some lacunas in the national literature on physical inactivity, sedentary behaviors, and unhealthy food habits in youth. First, a high concentration of studies focused on the South 
and Southeast regions of Brazil, whereas few included studies were conducted in North and Central-West regions (see Table 1). This characteristic has also been observed in other reviews of adolescent health ${ }^{84-86}$ and indicates that the development of scientific research related to the risk behaviors in Brazil has yet to cover all regions of the country. There is an effort of regional and national institutions to performed surveys involving adolescents from all regions of Brazil $^{15,26}$. However, public and private institutions should to invest more financially and academically in this research area, developing policies for the diagnosis and prevention of behavioral risk factors among young people from different regions in Brazil.

Another feature of this review reinforces the discrepancy between Brazilian regions in research on behavioral risk factors among Brazilian youth: only one included study was conducted in a lowHDI $(<0.600)$ site $^{34}$. The literature has pointed out the importance of assessing health-related risk behaviors in sites with low human development level because a lack of knowledge about health factors and structural problems in health systems available to the population of these sites contribute to the adoption of inappropriate behavior and the emergence of diseases in different age groups, including the young ${ }^{1,2}$. Therefore, institutions focused on population health should intensify their efforts to the diagnosis and treatment of behavioral risk factors among Brazilian youth from regions/sites lacking in research (Chart 1).

\section{Physical inactivity}

The inter-study comparison of physical inactivity prevalence has several limitations that should be considered when interpreting the results of this review. Firstly, the use of different instruments, recall time, and physical activity domains (i.e., total physical activity or leisure and transportation physical activity only) interferes

Chart 1. Summary of main findings from this review.

\begin{tabular}{|c|c|}
\hline Risk behaviors & Main findings \\
\hline $\begin{array}{l}\text { Physical } \\
\text { inactivity }\end{array}$ & $\begin{array}{l}\text { The prevalence of physical inactivity ranged from } 2.3 \% \text { to } 93.5 \% \text {. Only } 4 \text { studies } \\
(8.3 \%) \text { indicated physical inactivity prevalence of less than } 20 \% \text {, while } 28 \text { studies } \\
(58.3 \%) \text { estimated physical inactivity prevalences above } 50 \% \text {. } \\
\text { The } 95 \% \text { CIs for the prevalence of physical inactivity in boys and girls did not } \\
\text { overlap in } 87.5 \% \text { of studies. }\end{array}$ \\
\hline $\begin{array}{l}\text { Sedentary } \\
\text { behaviors }\end{array}$ & $\begin{array}{l}\text { Prevalence of high screen time use ranged from } 28.1 \% \text { to } 88.0 \% \text {, whereas the high } \\
\text { TV use ranged from } 11.3 \% \text { to } 79.5 \% \text {. Most of studies }(60 \%) \text { demonstrated } \\
\text { prevalence rates above } 50 \% \text {. } \\
\text {. The magnitude of difference between genders for the sedentary behaviors was small } \\
\text { at most of studies because the } 95 \% \text { CIs for the prevalence of sedentary behaviors in } \\
\text { boys and girls did not overlap in only three studies }(23.1 \%) \text {. }\end{array}$ \\
\hline $\begin{array}{l}\text { Unhealthy food } \\
\text { habits }\end{array}$ & $\begin{array}{l}\text { Overall, several indicators were used to identify unhealthy food habits. Some } \\
\text { criteria/studies have indicated estimates of unhealthy food habits in close to } 100 \% \text { of } \\
\text { adolescents. Moreover, it was consistent that low healthy food consumption was } \\
\text { more present than high unhealthy food consumption. } \\
\text { The high soft drink consumption was the outcome most frequently studied } \\
\text { regarding unhealthy food habits among Brazilian adolescents ( } 37.5 \% \text { of studies), } \\
\text { with prevalence rates ranged from } 20.4 \% \text { to } 71.0 \% \text {. The high sweets consumption } \\
\text { was evaluated in } 31.2 \% \text { of studies, and the prevalence rates ranged from } 20.1 \% \text { to } \\
96.9 \% \text {. } \\
\text { The prevalence of low fruit consumption among adolescents ranged from } 33.4 \% \text { to } \\
82.8 \% \text {, and the prevalence of low vegetable consumption ranged from } 36.3 \% \text { to } \\
75.8 \% \text {. Most studies ( } 60 \% \text { ) evaluating these risk behaviors has indicated prevalence } \\
\text { rates above } 50 \% \text {. } \\
\text { The magnitude of difference between genders for the prevalences of high unhealthy } \\
\text { food consumption or low healthy food consumption was discrete in most studies. }\end{array}$ \\
\hline
\end{tabular}


with the inter-study comparison. Secondly, the use of different cutoff points for the definition of physical inactivity is another important limitation of inter-study comparison (i.e., several physical inactivity definitions were adopted since the low frequency of participation in physical activities and sports until the estimating of the daily energy expenditure). Thirdly, different age groups were considered in the included studies - some studies involved early adolescents ${ }^{21,44,63,70}$, while others examined subjects in the late adolescence $^{19,20,25,35,62,61,66,72,78,79}$. Therefore, it is impossible to identify national and regional estimates of physical inactivity, or to analyze prevalence rates stratified by some study characteristics (e.g., survey periods or HDI level).

Despite these limitations for data analysis, this review provides a framework indicating that physical inactivity affects at least half of the adolescents in several sites of Brazil. Although there was a wide range in the prevalence of physical inactivity, a large number of studies found a physical inactivity prevalence above $50 \%$. Thus, studies consistently demonstrate that physical inactivity is a behavioral risk factor present in most adolescents and public policies should focus on active lifestyle promotion in youth.

The indication that physical inactivity affects more than half of young people was also found in adolescents from several countries, according to the GSHS ${ }^{13}$ data. This study also demonstrated that the percentage of physical inactivity exceeds $75 \%$ in more than half of the 34 surveyed countries, especially in girls ${ }^{13}$. The Health Behaviour in School-aged Children (HBSC) 2005-2006 survey ${ }^{87}$, which was an international study with adolescents from 41 countries in Europe and North America, also identified a physical inactivity prevalence above $50 \%$ in several countries. Thus, it is clear that physical inactivity is a worldwide phenomenon that has reached alarming levels in young people.

Some studies included in this review have identified possible exposure factors associated with physical inactivity among Brazilian adolescents. Several studies have found that female $15,17,20,24,25,33$, $34,35,39,45,46,60,63,66,79$ and older adolescents ${ }^{19,25,33,64,65}$, ${ }^{66,79}$ comprise population subgroups most exposed to physical inactivity. Other population subgroups have also been identified, including working adolescents ${ }^{19,79}$, those from the public school system ${ }^{15,62}$, and whose that parents are inactive ${ }^{18,63-65}$. Importantly, the association between socioeconomic status and physical activity varies; some studies highlight that adolescents of elevated socioeconomic status are at a higher risk for physical inactivity ${ }^{18,25,63}$, whereas others indicate an inverse association ${ }^{34,62,64}$.

The findings of these studies have contributed to the development of public policies promoting physical activity among Brazilian youth, based on the subgroups at higher risk for physical inactivity. However, future cross-sectional and longitudinal studies are necessary to identify others variables (e.g., socioeconomic conditions and parents' physical activity) associated with physical inactivity among Brazilian adolescents. Additionally, the study of variables from different levels, going from individual factors (e.g., satisfaction and self-efficacy for physical activity) to environmental contexts (e.g., favourable school and community environment for physical activity) is fundamental to determine the primary and different levels of the physical activity predictors.

In studies evaluating physical inactivity as an exposure factor and identifying health-related outcomes among Brazilian adolescents, we observed associations of physical inactivity with a greater prevalence of overweight/obesity $^{31,40,48,70,83}$, a high total cholesterol ${ }^{48}$, high blood pressure $^{41}$ and a high body fat percentage ${ }^{41,48}$. Finally, it has also been noted that the physical inactivity are associated with higher estimates of other risk behaviors, such as excessive time watching $\mathrm{TV}^{23,63}$ and the low consumption of fruits and/or vegetables ${ }^{32,46,83}$. The findings of these Brazilian studies have evidenced the association of physical inactivity and other health-related outcomes.

\section{Sedentary behaviors}

Although methodological differences are small in sedentary behavior studies when compared to studies evaluating physical activity, the inter-study comparison is also limited. First, the recall time to identify sedentary behaviors varied significantly, ranging from previous or habitual days to previous four months. The type of activities considered in the sedentary behavior also limited the comparison because some studies included TV use only and others also considered sedentary leisure habits (i.e., computer and video game use). However, the most important limitation was the use of different cutoff points for the determination of sedentary behavior, limiting the inter-study comparison (see Table 3).

This review demonstrates that most studies found a sedentary behavior prevalence above $50 \%$. Data obtained from the GSHS ${ }^{13}$ identified a 
high screen time use prevalence ( 3 or more hours per day) above $50 \%$ only in adolescents aged 13 to 15 years old from 4 of 34 countries (Cayman Islands, Colombia, Saint Lucia, and Seychelles). Additionally, higher prevalence rates have been found in countries of the Americas Region (not including Brazil in this study) ${ }^{13}$. The GSHS also showed that the prevalence of high screen time use were similar between the genders in most countries.

Data obtained from the Youth Risk Behavior Surveillance (YRBS) survey ${ }^{12}$, conducted with American adolescents in 2009, also show higher estimates of sedentary behavior $(24.9 \%$ and $32.8 \%$ of adolescents use computer/videogames and watch TV three or more hours per day, respectively). However, the sedentary behavior prevalence among American adolescents was still below those found in most Brazilian adolescent studies. Therefore, it is clear that sedentary behavior is a behavioral risk factor present in a large proportion of Brazilian adolescents. Preventive measures should be taken to reduced this risk behavior and improve health in Brazilian youth.

The literature provides little evidence regarding factors associated with the adoption of a sedentary lifestyle among Brazilian adolescents. Gender was associated with sedentary behaviors in a few studies ${ }^{22,46}$. In addition, a few studies identified an association between sedentary behaviors and other sociodemographic variables, such as socioeconomic status ${ }^{22}$, parental education ${ }^{65,82}$, age $^{19,82}$, and the type of school ${ }^{15}$. Thus, study is required to evaluate potential factors associated with sedentary behavior during adolescence because these factors are not well understood in the Brazilian youth-related literature. Longitudinal monitoring of this risk behavior during adolescence and the evaluation of predictors from different levels, as specified for studies of physical inactivity, are also fundamental to understanding the reasons for the high sedentary behavior estimate among Brazilian adolescents.

\section{Unhealthy food habits}

The inter-study comparison of the prevalence of unhealthy food habits should also be performed with caution. The amount and types of food included in the questionnaires (e.g., some studies considered natural fruit juice for fruit consumption definition, whereas others do not) has significant variations between studies. The use of different cutoff points (e.g., the cutoff points for identifying the low consumption of fruits and vegeta- bles ranged from four times per week to five portions per day) is also an important limitation of the inter-study comparison. Therefore, the analysis of the results of unhealthy food habits should consider these characteristics.

Based on the results of the studies included in this review, a high number of adolescents in several Brazilian sites are exposed to unhealthy food habits, with some studies estimating rates of unhealthy food habits in close to $100 \%^{20,49,54,55}$ of adolescents. Most studies also demonstrate a prevalence of low healthy food consumption that was higher than the prevalence of high unhealthy food consumption ${ }^{15,24,46,50,54,61,66,69,74-76}$. These results indicate a need to promote proper eating habits, and such healthy diet policies should primarily encourage the consumption of healthy foods.

Estimates of unhealthy food habits in several included studies are very close to those found in developed countries like the United States ${ }^{12}$ and Canada $^{87}$, where health problems related to a poor diet (especially high blood pressure and overweight/obesity) are already well established among the younger population. Recent data from the YRBS ${ }^{12}$ indicate that $29 \%$ of American adolescents consume soft drinks daily. Several Brazilian studies have found a prevalence of high soft drink consumption very close to those obtained from American adolescents, including studies that used the same cutoff point (one or more times per day $)^{50,61,77}$. If interventions are not developed to reduce these behaviors, serious health problems (such as type 2 diabetes, high blood pressure, and dyslipidemia) may reaching alarming proportions in Brazilian youth and worsen the NCD framework in Brazil.

Some studies have identified factors associated with unhealthy food habits among Brazilian adolescents. Although some studies show significant differences between genders in unhealthy food habits ${ }^{20,24,66,79}$, the direction of this association is inconsistent and varies according to the unhealthy food habit outcome. These features were also observed in this review (see Table 4). Other studies have found that low socioeconomic status is associated with a higher prevalence of low healthy food consumption (e.g., fruits and vegetables $)^{26,70,75}$, but the direction of the association between these variables was not consistent between studies. These findings confirmed that behavioral characteristics and associated factors may vary from region to region, even within the same country. Therefore, we point out the importance of considering local and regional Brazilian adolescents peculiarities to understand the main var- 
iables associated with high unhealthy food habits estimates. Additionally, these peculiarities should be considered when determining the primary focuses of healthy eating interventions.

Only two studies ${ }^{69,76}$ included in this review found a significant association between unhealthy food habits and overweight/obesity. No study identified a significant association between unhealthy food habits and other health outcomes in adolescence (e.g., high total cholesterol or high blood pressure). Two features of this review may be related to these results. First, most Brazilian studies that evaluated food habits and metabolic health-related risk factors (e.g., low HDL, high cholesterol, high blood pressure) adopted nonprobabilistic methods for the sample selection and in turn were not included in this review. Moreover, some studies evaluating the association between food habits and health outcomes may have been excluded for having a more complete replication study, considering the purposes of this review.

However, lack of evidence for an association between unhealthy food habits and health outcomes may be related to another factor. Most studies included in this review evaluated food habits using a food-frequency questionnaire without portion sizes. The quantification of total intake based on frequency alone may be inaccurate because of the absence of information on portion sizes ${ }^{87}$. These characteristics have been identified as the reason for the lack of association between unhealthy food habits and overweight/ obesity in studies of the $\mathrm{HBSC}^{87}$. Therefore, the use of more precise methods to determine food habits in adolescence is fundamental to identify the relationship between food habits and health outcomes.

\section{Final considerations}

Some limitations of this review should be highlighted. The first limitation was discussed previously and is related to the viability of inter-study comparison on the prevalence of physical inactivity, sedentary behaviors, and unhealthy food habits. A second limitation is related to the possibility of that some important studies have not been included in this review. A large number of adolescent-related health behavior studies are theses and dissertations, and the time to publish some manuscripts in peer-reviewed literature is often lengthy. However, it is believed that the main research on physical inactivity, sedentary behav- iors, and unhealthy food habits among Brazilian adolescents, including the Pesquisa Nacional de Saúde do Escolar ${ }^{15,26}$, were presented in this review. Third, this review was limited to those studies that presented data on the prevalence of at least one of three behavioral risk factors studied, not including studies that have addressed these behaviors with other methods of analysis (e.g., mean time watching TV or daily mean of servings of fruits and vegetables).

This systematic review demonstrates that three important behavioral risk factors are present in a large proportion of Brazilian youth. As such, this review has identified important determinants of the present and future health of this population. The estimates of these risk behaviors among Brazilians adolescents were very close to or even greater than those found in developed countries in several studies. Considering that NCDs are the leading causes of death in Brazilian adults, if public policies to combat these NCD behavioral risk factors during youth are not developed, future estimates may be even more worrisome. Further studies identifying and tracking the possible factors associated with the adoption of NCD behavioral risk factors in adolescence are required, and we highlight the urgent need for interventions to promote health among Brazil youth.

\section{Collaborations}

V. C. Barbosa Filho participated in all stages of the study, including the search and review of studies, analysis and writing of the manuscript. W. de Campos and A. S. Lopes participated in the structuring of the methods, analysis of results and critical review of the manuscript.

\section{Acknowledgments}

The first author was supported by the Coordenação de Aperfeiçoamento de Pessoal de Nível Superior (CAPES) during the Master's scholarship. We thank the reviewers for their constructive contributions. 


\section{References}

1. World Health Organization (WHO). 2008-2013. Action plan for the global strategy for the prevention and control of noncommunicable diseases. Geneva: World Health Organization; 2008.

2. World Health Organization WHO). Global status report on noncommunicable diseases 2010. Geneva: World Health Organization; 2011.

3. Cecchini M, Sassi F, Lauer JA, Lee YY, GuajardoBarron V, Chisholm D. Tackling of unhealthy diets, physical inactivity, and obesity: health effects and cost-effectiveness. Lancet 2010; 376(9754):1775-1784.

4. World Health Organization (WHO). Global recommendations on physical activity for health. Geneva: World Health Organization; 2010.

5. World Health Organization (WHO). Global health risks: mortality and burden of disease attributable to selected major risks. Geneva: World Health Organization; 2009.

6. World Health Organization (WHO). Global strategy on diet, physical activity and health. Geneva: World Health Organization; 2004.

7. Dunstan DW, Barr ELM, Healy GN, Salmon J, Shaw JE, Balkau B, Magliano DJ, Cameron AJ, Zimmet $\mathrm{PZ}$, Owen N. Television viewing time and mortality: the Australian Diabetes, Obesity and Lifestyle Study (AusDiab). Circulation 2010; 121:384-391.

8. Katzmarzyk PT, Church TS, Craig CL, Bouchard C. Sitting time and mortality from all causes, cardiovascular disease, and cancer. Med Sci Sports Exerc 2009; 41(5):998-1005.

9. Stamatakis E, Hamer M, Dunstan DW. Screen-based entertainment time, all-cause mortality, and cardiovascular events: population-based study with ongoing mortality and hospital events follow-up. $J$ Am Coll Cardiol 2011;57:292-299.

10. Thorp AA, Owen N, Neuhaus M, Dunstan DW. Sedentary behaviors and subsequent health outcomes in adults a systematic review of longitudinal studies, 1996-2011. Am J Prev Med 2011; 41(2):207215 .

11. Hancox RJ, Milne BJ, Poulton R. Association between child and adolescent television viewing and adult health: a longitudinal birth cohort study. Lancet 2004; 364(9430):257-262.

12. Eaton D, Kann L, Kinchen S, Shanklin S, Ross J, Hawkins J, Harris WA, Lowry R, McManus T, Chyen $\mathrm{D}$, Lim C, Whittle L, Brener ND, Wechsler H. Youth Risk Behavior Surveillance - United States, 2009. MMWR Surveill Summ 2010; 59(05):1-142.

13. Guthold R, Cowan MJ, Autenrieth CS, Kann L, Riley LM. Physical activity and sedentary behavior among schoolchildren: a 34-country comparison. $J$ Pediatr 2010; 157(1):43-49.

14. World Health Organization (WHO). Global schoolbased student health survey (GSHS). [artigo da internet] 2011. [acessado 2011 jun 15]. Disponível em: http://www.who.int/chp/gshs/en/.

15. Malta DC, Sardinha LMV, Mendes I, Barreto SM, Giatti L, Castro IRR, Moura L, Dias AJR, Crespo C. Prevalência de fatores de risco e proteção de doenças crônicas não transmissíveis em adolescentes: resultados da Pesquisa Nacional de Saúde do Escolar (PeNSE), Brasil, 2009. Cien Saude Coletiva 2009; 15(Supl. 2):3009-3019.
16. Tassitano RM, Barros MVG, Tenório MCM, Bezerra J, Florindo AA, Reis RS. Enrollment in physical education is associated with health-related behavior among high school students. J Sch Health 2010; 80(3):126-133.

17. Fermino RC, Rech CR, Hino AAF, Rodriguez-Añez CR, Reis RS. Physical activity and associated factors in high-school adolescents in Southern Brazil. Rev Saude Publica 2010; 44(6):986-995.

18. Dumith SC, Domingues MR, Gigante DP, Hallal PC, Menezes AMB, Kohl HW. Prevalence and correlates of physical activity among adolescents from Southern Brazil. Rev Saude Publica 2010; 44(3):457-467.

19. Tenório MCM, Barros MVG, Tassitano RM, Bezerra J, Tenório JM, Hallal PC. Atividade física e comportamento sedentário em adolescentes estudantes do ensino médio. Rev Bras Epidemiol 2010; 13(1):105117.

20. Beck CC, Lopes AS, Giuliano ICB, Borgatto AF. Fatores de risco cardiovascular em adolescentes de município do sul do Brasil: prevalência e associações com variáveis sociodemográficas. Rev Bras Epidemiol 2011; 14(1):36-49.

21. Bergmann MLA, Bergmann GG, Halpern R, Rech RR, Constanzi CB, Alli LR. Associated factors to total cholesterol: school-based study in Southern Brazil. Arq Bras Cadiol 2011; 97(1):17-25.

22. Dumith SC, Hallal PC, Menezes AMB, Araújo CL. Sedentary behavior in adolescents: the 11-year follow-up of the 1993 Pelotas (Brazil) birth cohort study. Cad Saude Publica 2010; 26(10):1928-1936.

23. Silva KS, Nahas MV, Hoefelmann LP, Lopes AS, Oliveira ES. Associação entre atividade física, índice de massa corporal e comportamentos sedentários em adolescentes. Rev Bras Epidemiol 2008; 11(1):159-168.

24. Castro IRR, Cardoso LO, Engstrom EM, Levy RB, Monteiro CA. Vigilância de fatores de risco para doenças não transmissíveis entre adolescentes: a experiência da cidade do Rio de Janeiro, Brasil. Cad Saude Publica 2008; 24(10):2279-2288.

25. Ceschini FL, Andrade DR, Oliveira LC, Araújo Júnior JF, Matsudo VKR. Prevalence of physical inactivity and associated factors among high school students from state's public schools. J Pediatr (Rio J) 2009; 85(4):301-306.

26. Levy RB, Castro IRR, Cardoso LO, Tavares LF, Sardinha LMV, Gomes FS, Costa AWN. Consumo e comportamento alimentar entre adolescentes brasileiros: Pesquisa Nacional de Saúde do Escolar (PeNSE), 2009. Cien Saude Coletiva 2009; 15(Supl. 2):3085-3097.

27. Nahas MV, Barros MVG, Goldfine BD, Lopes AS, Hallal PC, Farias Júnior JC, Oliveira ES. Physical activity and eating habits in public high schools from different regions in Brazil: the Saude na Boa project. Rev Bras Epidemiol 2009; 12(2):270-277.

28. Magalhães VC, Mendonça GAS. Prevalência e fatores associados a sobrepeso e obesidade em adolescentes de 15 a 19 anos das regiões Nordeste e Sudeste do Brasil, 1996 a 1997. Cad Saude Publica 2003; 19(Supl. 1):129-139. 
29. Enes CC, Pegolo GE, Silva MV. Influência do consumo alimentar e do padrão de atividade física sobre o estado nutricional de adolescentes de Piedade, São Paulo. Rev Paul Pediatr 2009; 27(3):265-271.

30. Rivera IR, Silva MAM, Silva RDTA, Oliveira BAV, Carvalho ACC. Physical inactivity, TV-watching hours and body composition in children and adolescents. Arq Bras Cardiol 2010; 95(2):159-165.

31. Frainer DES, Silva MCM, Santana MLP, Santos NS, Oliveira LPM, Barreto ML, Assis AMO. Prevalence and associated factors of overweight in adolescents from Salvador, Bahia, Brazil. Rev Bras Med Esporte 2011; 17(2):102-106.

32. Farias Júnior JC, Mendes JKF, Barbosa DBM. Associação entre comportamentos de risco à saúde em adolescentes. Rev Bras Cineantropom Desempenho Hum 2007; 9(3):250-258.

33. Souza GS, Duarte MFS. Estágios de mudança de comportamento relacionados à atividade física em adolescentes. Rev Bras Med Esporte 2005; 11(2):104108.

34. Silva DAS, Smith-Menezes A, Almeida-Gomes M, Sousa FD. Estágios de mudanças de comportamento para atividade física em estudantes de uma cidade do Brasil. Rev Salud Publica 2010; 12(4):623-634.

35. Costa MCO, Silva MCM, Santos JS, Teles C, Souza KEP, Melo BO. Estilo de vida de adolescentes: consumo alimentar, de bebida alcoólica e atividade física em Teixeira de Freitas/Bahia. Rev Baiana Saude Publica 2004; 28(2):151-166.

36. Oliveira TC, Silva AAM, Santos CJN, Silva JS, Conceição SIO. Atividade física e sedentarismo em escolares da rede pública e privada de ensino em São Luís. Rev Saude Publica 2010; 44(6):996-1004.

37. Borges CQ, Silva RCR, Assis AMO, Pinto EJ, Fiaccone RL, Pinheiro SMC. Fatores associados à anemia em crianças e adolescentes de escolas públicas de Salvador, Bahia, Brasil. Cad Saude Publica 2009; 25(4):877-888.

38. Conceição SIO, Santos CJN, Silva JS, Oliveira TC. Consumo alimentar de escolares das redes pública e privada de ensino em São Luís, Maranhão. Rev Nutr 2010; 23(6):993-1004.

39. Farias Júnior JC, Lopes AS, Mota J, Santos MP, Ribeiro JC, Hallal PC. Perception of the social and built environment and physical activity among Northeastern Brazil adolescents. Prev Med 2011; 52(2):114-119.

40. Souza CO, Silva RCR, Assis AMO, Fiaccone RL, Pinto EJ, Moraes LTLP. Association between physical inactivity and overweight among adolescents in Salvador, Bahia-Brazil. Rev Bras Epidemiol 2010; 13(3): 468-475.

41. Griz LHM, Viégas M, Barros M, Griz AL, Freese E, Bandeira F. Prevalence of central obesity in a large sample of adolescents from public schools in Recife, Brazil. Arq Bras Endocrinol Metab 2010; 54(7):607611.

42. Souza DPO, Silveira Filho DX. Uso recente de álcool, tabaco e outras drogas entre estudantes adolescentes trabalhadores e não trabalhadores. Rev Bras Epidemiol 2007; 10(2):276-287.

43. Pivetta LA, Gonçalves-Silva RMV. Compulsão alimentar e fatores associados em adolescentes de Cuiabá, Mato Grosso, Brasil. Cad Saude Publica 2010; 26(2):337-346.
44. Cândido APC, Benedetto R, Castro APP, Carmo JS, Nicolato RLC, Nascimento-Neto, RM, Freitas RN, Freitas SN, Caiaffa WT, Machado-Coelho GLL. Cardiovascular risk factors in children and adolescents living in an urban area of Southeast of Brazil: Ouro Preto Study. Eur J Pediatr 2009; 168(11):1373-1382.

45. Silva RCR, Malina RM. Nível de atividade física em adolescentes do município de Niterói, Rio de Janeiro, Brasil. Cad Saude Publica 2000; 16(4):1091-1097.

46. Fernandes RA, Christofaro DGD, Casonatto J, Kawaguti SS, Ronque VER, Cardoso JR, Freitas Junior IF, Oliveira AR. Cross-sectional association between healthy and unhealthy food habits and leisure physical activity in adolescents. J Pediatr (Rio J) 2011; 87(3):252-256.

47. Gomes VB, Siqueira KS, Sichieri R. Atividade física em uma amostra probabilística da população do município do Rio de Janeiro. Cad Saude Publica 2001; 17(4):969-976.

48. Ribeiro RQC, Lotufo PA, Lamounier JA, Oliveira RG, Soares JF, Botter DA. Additional cardiovascular risk factors associated with excess weight in children and adolescents. The Belo Horizonte Heart Study. Arq Bras Cardiol 2006; 86(6):408-418.

49. Andrade SCD, Barros MBA, Carandina L, Goldbaum M, Fisberg RM, Cesar CLG, Fisberg RM. Dietary Quality Index and associated factors among adolescents of the state of Sao Paulo, Brazil. J Pediatr 2010; 156(3):456-460.

50. Gurgel CV, Rios D, Oliveira TM, Tessarolli V, Carvalho FP, Machado MAAM. Risk factors for dental erosion in a group of 12- and 16-year-old Brazilian schoolchildren. Int J Paediatr Dent 2011; 21(1):50-57.

51. Teixeira MH, Veiga GV, Sichieri R. Consumo de gordura e hipercolesterolemia em uma amostra probalibística de estudantes de Niterói, Rio de Janeiro. Arq Bras Endocrinol Metab 2007; 51(1):65-71.

52. Nogueira FAM, Sichieri R. Associação entre consumo de refrigerantes, sucos e leite, com o índice de massa corporal em escolares da rede pública de Niterói, Rio de Janeiro, Brasil. Cad Saude Publica 2009; 25(12):2715-2724.

53. Carmo MB, Toral N, Silva MV, Slater B. Consumo de doces, refrigerantes e bebidas com adição de açúcar entre adolescentes da rede pública de ensino de Piracicaba, São Paulo. Rev Bras Epidemiol 2006; 9(1): 121-130.

54. Toral N, Slater B, Silva MV. Consumo alimentar e excesso de peso de adolescentes de Piracicaba, São Paulo. Rev Nutr 2007; 20(5):449-459.

55. Bigio RS, Verly Júnior E, Castro MA, Cesar CLG, Fisberg RM, Marchioni DML. Determinants of fruit and vegetable intake in adolescents using quantile regression. Rev Saude Publica 2011; 45(3):448-456.

56. Romero A, Slater B, Florindo AA, Latorre MRDO, Cezar C, Silva MV. Determinantes do índice de massa corporal em adolescentes de escolas públicas de Piracicaba, São Paulo. Cien Saude Coletiva 2010; 15(1):141-149.

57. Abreu MNS, Souza CF, Caiaffa WT. Tabagismo entre adolescentes e adultos jovens de Belo Horizonte, Minas Gerais, Brasil: influência do entorno familiar e grupo social. Cad Saude Publica 2011; 27(5):935-943. 
58. Anteghini M, Fonseca $\mathrm{H}$, Ireland $\mathrm{M}$, Blum RW Health risk behaviors and associated risk and protective factors among Brazilian adolescents in Santos, Brazil. J Adol Health 2001; 28(4):295-302.

59. Madruga SW, Araújo CL, Bertoldi AD. Frequency of fiber-rich food intake and associated factors in a Southern Brazilian population. Cad Saude Publica 2006; 25(10):2249-2259.

60. Gordia AP, Quadros TMB, Campos W, Petroski EL. Nível de atividade física em adolescentes e sua associação com variáveis sociodemográficas. Rev Port Cien Desp 2010; 10(1):172-179.

61. Legnani E, Legnani RFS, Barbosa Filho VC, Gasparotto GS, Campos W, Lopes AS. Fatores de risco à saúde cardiovascular em escolares da Tríplice Fronteira. Motriz: Rev Educ Fís 2011;17(4):640-649.

62. Moraes ACF, Fernandes CAM, Elias RGM, Nakashima ATA, Reichert FF, Falcão MC Prevalence of physical inactivity and associated factors among adolescents. Rev Assoc Med Bras 2009; 55(4):523-528.

63. Hallal PC, Bertoldi AD, Gonçalves H, Victora CG. Prevalência de sedentarismo e fatores associados em adolescentes de 10-12 anos de idade. Cad Saude Publica 2006; 22(6):1277-1287.

64. Bertoni N, Bastos FI, Mello MB, Makuch MY, Sousa $\mathrm{MH}$, Osis MJ, Faundes A. Uso de álcool e drogas e sua influência sobre as práticas sexuais de adolescentes de Minas Gerais, Brasil. Cad Saude Publica 2009; 25(6):1350-1360.

65. Vasques DG, Lopes AS. Fatores associados à atividade física e aos comportamentos sedentários em adolescentes. Rev Bras Cineantropom Desempenho Hum 2009; 11(10):59-66.

66. Farias Júnior JC. Comportamentos de risco relacionados à saúde em adolescentes. Rev Bras Cien Mov 2004; 12(1):7-12.

67. Malcon MC, Menezes AMB, Chatkin M. Prevalência e fatores de risco para tabagismo em adolescentes. Rev Saude Publica 2003; 37(1):1-7.

68. Strauch ES, Pinheiro RT, Silva RA, Horta BL. Alcohol use among adolescents: a population-based study. Rev Saude Publica 2009; 43(4):647-655.

69. Campagnolo PDB, Vitolo MR, Gama CM, Stein AT. Prevalence of overweight and associated factors in southern Brazilian adolescents. Public Health 2008; 122(5):509-515.

70. Suñe FR, Dias-da-Costa JS, Olinto MTA, Pattussi MP. Prevalência e fatores associados para sobrepeso e obesidade em escolares de uma cidade no Sul do Brasil. Cad Saude Publica 2007; 23(6):1361-1371.

71. Cimadon HMS, Geremia R, Pellanda LC. Dietary habits and risk factors for atherosclerosis in students from Bento Gonçalves (state of Rio Grande do Sul). Arq Bras Cardiol 2010; 95(2):166-172.

72. Terres NG, Pinheiro RT, Horta BL, Pinheiro KAT, Horta LL. Prevalência e fatores associados ao sobrepeso e à obesidade em adolescentes. Rev Saude Publica 2006; 40(4):627-633.

73. Dutra CL, Araújo CL, Bertoldi AD. Prevalência de sobrepeso em adolescentes: um estudo de base populacional em uma cidade no Sul do Brasil. Cad Saude Publica 2006; 22(1):151-162.

74. Arruda ELM, Lopes AS. Hábitos alimentares de adolescentes da região serrana de Santa Catarina, Brasil. Rev Bras Cineantropom Desempenho Hum 2007; 9(1):5-11.
75. Neutzling MB, Araújo CLP, Vieira MFA, Hallal PC, Menezes AMB. Frequência de consumo de dietas ricas em gordura e pobres em fibra entre adolescentes. Rev Saude Publica 2007; 41(3):336-342.

76. Barbiero SM, Pellanda LC, Cesa CC, Campagnolo P, Beltramo F, Abrantes CC. Overweight, obesity and other risk factors for IHD in Brazilian schoolchildren. Public Health Nutr 2008; 12(5):710-715.

77. Costa MCD, Cordoni Júnior L, Matsuo T. Hábito alimentar de escolares adolescentes de um município do oeste do Paraná. Rev Nutr 2007; 20(5):461471.

78. Romanzini M, Reichert FF, Lopes AS, Petroski EL, Farias Júnior JC. Prevalência de fatores de risco cardiovascular em adolescentes. Cad Saude Publica 2008; 24(11):2573-2581.

79. Farias Júnior JC, Nahas MV, Barros MVG, Loch MR, Oliveira ESA, Bem MFL, Lopes AS. Comportamentos de risco à saúde em adolescentes no Sul do Brasil: prevalência e fatores associados. $\mathrm{Rev} \mathrm{Pa}$ nam Salud Publica 2009; 25(4):344-352.

80. Neutzling MB, Assunção MCF, Malcon MC, Hallal PC, Menezes AMB. Hábitos alimentares de escolares adolescentes de Pelotas, Brasil. Rev Nutr 2010; 23(3):379-388.

81. Tavares BF, Béria JU, Lima MSD. Factors associated with drug use among adolescent students in Southern Brazil. Rev Saude Publica 2004; 38(6):787-796.

82. Campagnolo PDB, Vitolo MR, Gama CM. Fatores associados ao hábito de assistir TV em excesso entre adolescentes. Rev Bras Med Esporte 2008; 14(3):197-200.

83. Silva KS, Nahas MV, Peres KG, Lopes AS. Fatores associados à atividade física, comportamento sedentário e participação na Educação Física em estudantes do Ensino Médio em Santa Catarina, Brasil. Cad Saude Publica 2009; 25(10):2187-2200.

84. Dumith SC. Physical activity in Brazil: a systematic review. Cad Saude Publica 2009; 25(Supl. 3):415-426.

85. Hallal PC, Dumith SC, Bastos JP, Reichert FF, Siqueira FV, Azevedo MR. Evolução da pesquisa epidemiológica em atividade física no Brasil: revisão sistemática. Rev Saude Publica 2007; 41(3):453-460.

86. Souza EA, Barbosa Filho VC, Nogueira JAD, Azevedo MR. Physical activity and healthy eating in Brazilian students: a review of intervention programs. Cad Saude Publica 2011; 27(8):1459-1471.

87. Haug E, Rasmussen M, Samdal O, Iannotti R, Borraccino A, Vereecken C, Melkevik O, Lazzeri G, Giacchi M, Ercan O, Due P, Ravens-Sieberer U, Currie C, Morgan A, Ahluwalia N. Overweight in school-aged children and its relationship with demographic and lifestyle factors: results from the WHO-Collaborative Health Behaviour in Schoolaged Children (HBSC) Study. Int J Public Health 2009; 54(Supl. 1):167-179.

Artigo apresentado em 15/03/2012

Aprovado em 02/04/2012

Versão final apresentada em 11/04/2012 
